\title{
LncRNA NKX2-1-AS1 As ceRNA Promotes Tumor Progression and Angiogenesis by Upregulating SERPINE1 Expression and Activating VEGFR-2 Signaling Pathway in Gastric Cancer
}

Fei Teng

Minhang Hospital, Fudan University

Juxiang Zhang

Shanghai Jiao Tong University

Yi Chen

Minhang Hospital, Fudan University

Xiaodong Shen

Minhang Hospital, Fudan University

Yanjiao Guo

Minhang Hospital, Fudan University

Puhua Wang

Minhang Hospital, Fudan University

Ming Lei

Minhang Hospital, Fudan University

Yiou Cao

Minhang Hospital, Fudan University

Shao-Qun Liu ( $\nabla$ liushaoqun@fudan.edu.cn )

Minhang Hospital of Fudan University https://orcid.org/0000-0002-5671-3574

Research

Keywords: Gastric cancer, NKX2-1 antisense RNA 1, competing endogenous RNA, serpin family E member 1, VEGFR-2 signaling pathway

Posted Date: August 1st, 2020

DOI: https://doi.org/10.21203/rs.3.rs-48450/v1

License: (c) (1) This work is licensed under a Creative Commons Attribution 4.0 International License.

Read Full License 

LncRNA NKX2-1-AS1 as ceRNA promotes tumor progression and angiogenesis by upregulating SERPINE1 expression and activating VEGFR-2 signaling pathway in gastric cancer

Fei Teng ${ }^{1,2} \dagger$, Ju-Xiang Zhang ${ }^{3 \dagger}$, Yi Chen ${ }^{1,2}$, Xiao-Dong Shen ${ }^{1,2}$, Yan-Jiao Guo ${ }^{1,2}$, PuHua Wang ${ }^{1,2}$, Ming Lei ${ }^{1,2}$, Yi-Ou Cao ${ }^{1,2^{*}}$, Shao-Qun Liu ${ }^{1,2 *}$

*Correspondence: liushaoqun@fudan.edu.cn; caoyiou@fudan.edu.cn

${ }^{1}$ Department of Gastrointestinal Surgery, Minhang Hospital, Fudan University, Shanghai, 201199, P. R. China.

${ }^{2}$ Institute of Fudan-Minhang Academic Health System, Minhang Hospital, Fudan University, Shanghai, 201199, P. R. China.

${ }^{3}$ Shanghai Med-X Engineering Center for Medical Equipment and Technology, School of Biomedical Engineering, Shanghai Jiao Tong University, Shanghai 200030, P. R. China

$\dagger$ Fei Teng and Ju-Xiang Zhang contributed equally to this work.

Full list of author information is available at the end of the article

Word count: 8258 
Abstract

Background: Recent evidence indicated that the lncRNA NKX2-1-AS1 (NKX2-1 antisense RNA 1) plays an important role in cancer progression and metastasis. However, the associated molecular mechanisms of NKX2-1-AS1 in GC are still unclear.

Methods: To determine the target of the study by bioinformatic analysis. NKX21-AS1 expression was measured in paired tumor and non-tumor tissues of $178 \mathrm{GC}$ patients, by quantitative reverse transcription PCR. The in vitro and in vivo biological functions of NKX2-1-AS1 were examined by loss-of-function and gain-of-function experiments. The potential mechanisms of this competing endogenous RNA (ceRNA) were elucidated using dual-luciferase reporter assay, quantitative PCR, Western blot, and fluorescence in situ hybridization (FISH).

Results: NKX2-1-AS1 expression was upregulated in GC cell lines and tumor tissues, which was correlated with tumor progression and enhanced angiogenesis. Functionally, NKX2-1-AS1 overexpression promoted GC cell proliferation, metastasis, invasion, and angiogenesis, while NKX2-1-AS1 downregulation reversed these effects, both in vitro and in vivo. Bioinformatics analysis and dual-luciferase assay showed that the microRNA miR-145-5p is a direct target of NKX2-1-AS1, and that NKX2-1-AS1 acts as a ceRNA that regulates angiogenesis in the context of GC. The mechanistic study revealed that miR-145-5p specifically targets serpin family E member 1 (SERPINE1), and that the complex NKX2-1-AS1/miR-145-5p activates VEGFR2 signaling, via SERPINE1, to promote tumor proliferation and angiogenesis.

Conclusion: NKX2-1-AS1 upregulation is associated with tumor cell proliferation, increased angiogenesis, and poor prognosis in GC patients. NKX2-1-AS1 regulates SERPINE1 expression and VEGFR2 signaling by acting as a ceRNA for miR-145-5p.

Keywords: Gastric cancer; NKX2-1 antisense RNA 1; competing endogenous RNA; serpin family E member 1; VEGFR-2 signaling pathway 


\section{Background}

Gastric cancer (GC) is the fourth most common cancer and the third most common cause of cancer-related deaths worldwide. GC prevalence is higher in East Asia, namely in China and Japan (1). Due to the lack of early symptoms, GC diagnosis is often accompained by late stage disease (2). Despite the significant improvement in GC diagnosis and treatment in the recent decades, the prognosis of patients with advance disease is still highly unfavorable $(3,4)$. Currently, GC-associated tumor angiogenesis is regarded as a main cause of unfavorable patient outcomes (5). Therefore, understanding the molecular mechanisms of tumor angiogenesis is key to limit GC progression and improve patient diagnosis and outcome for the improvement of GC progression, diagnosis, and prognosis.

Long non-coding RNAs (lncRNAs) are a class of RNA polymerase II transcripts of $>200$ nucleotides long (6). Increasing evidence in recent years demonstrated that lncRNAs possess numerous biological functions and are involved in the regulation of multiple processes in disease, especially in cancer $(7,8)$. Unlike microRNAs (miRNAs), the unifying molecular mechanism for lncRNAs has not yet been reported (9). However, studies have shown that some lncRNAs can "sponge" and competitively inhibit the biological functions of miRNAs $(10,11)$. The lncRNA NKX2-1-AS1 was first reported to be highly expressed in primary lung adenocarcinomas, compared to squamous carcinomas, and to be associated with lung carcinoma cell migration (12). Bioinformatic studies also revealed that NKX2-1-AS1 may act as a ceRNA in GC (13). Still, changes in NKX2-1-AS1 expression, and its role in cancer is largely unknown. Accordingly, its functions and potential regulatory mechanism in GC have not been investigated.

The plasminogen activation system, which consists in the urokinase-type plasminogen activator (uPA), the cellular receptor for uPA (uPAR), and its specific inhibitor plasminogen activator inhibitor-1 (PAI-1 or SERPINE1), plays a crucial role in tumor progression and angiogenesis $(14,15)$. Numerous studies demonstrated that SERPINE1 overexpression is associated with the tumor progression and unfavorable 
outcome in several cancer models, including GC $(16,17)$. Several mechanisms have been proposed that explain the correlation of SERPINE1 with cancer progression. In particular, the role of SERPINE1 in tumor angiogenesis has been intensively investigated. Studies with SERPINE1-deficient (SERPIN1 ${ }^{-/}$) mice showed that host SERPINE1 expression is essential for tumor angiogenesis (18-20), and that its effects are likely mediated through the modulation of endothelial cell plasmin-mediated proteolysis (21), migration $(22,23)$, and apoptosis (24). Recently, it was demonstrated that the specific inhibition of SERPINE1 by the specific PAL-1 inhibitor SK-216 limited tumor angiogenesis in vivo and suppressed VEGF-induced migration and tube formation by human umbilical vein endothelial cells (HUVECs) in vitro (25). Based on these findings, it is speculated that the association between SERPINE1 overexpression and GC progression results from increased tumor angiogenesis promoted by SERPINE1. 
Methods:

Download of RNA-seq data of GC patients from The Cancer Genome Atlas (TCGA)

A comprehensive analysis was performed on all GC patients with RNA-seq data obtained from TCGA (www.portal.gdc.cancer.gov). Patients were screened based on 1) Stomach adenocarcinoma (STAD); 2) Sufficient data for analysis; 3) Absence of other malignancies; 4) Not having received radiation or chemotherapy before surgery. A total of 373 samples consisting of 343 tumor tissues and 30 adjacent non-cancerous tissues were included in this analysis. This study was conducted in accordance with the publication guidelines provided by the TCGA (www.cancergenome.nih.gov/publications/publicationlines).

RNA-seq data and differential gene expression analysis

The RNA expression profiles (level 3) of the STAD patients were obtained from TCGA-STAD (dated back to Sep. 1, 2017). This database provides normalized transcriptomic data from high-throughput sequencing, including lncRNA, mRNA, and miRNA profiles. Each sample consisted in the corresponding RNAseq, miRNAseq, and clinical data. Differential lncRNA, mRNA and miRNA expression between tumor and adjacent non-tumor tissues was analyzed using the edgeR package (26) in R (version 3.4.3, 2017, https://www.r-project.org). lncRNAs and mRNAs showing a log fold change (FC) of $>2.0$ and a false discovery rate (FDR)-adjusted $\mathrm{P}$ value of $<0.01$, were considered to be differentially expressed (DElncRNA and DEmRNA, respectively). Differentially expressed miRNAs (DEmiRNA) were identified by a $\log (\mathrm{FC})>1.5$ and FDR-adjusted P value of $<0.01$. Heatmaps and volcano plots of DElncRNA, DEmRNA and DEmiRNA were generated using the "pheatmap" and "ggplot2" packages in R (27). KEGG pathway enrichment analysis was performed on DEmRNAs using “clusterProfiler" and visualized by "ggplot2". Affected pathways were identified by P $<0.001$ and fold enrichment $>1.5$. The effect of differentially expressed RNAs on patient survival was analyzed by "survival" and "q-value" at $\mathrm{P}<0.05$. 


\section{Construction of the ceRNA network}

To determine lncRNA-miRNA-mRNA interactions, we considered only the pool of dysregulated lncRNAs and mRNAs. Then, we identified the miRNA seed sequences that function as shared (i.e, overlapping) binding sites for these lncRNAs and mRNAs. LncRNA-miRNA interaction was predicted by miRcode (www.mircode.org). miRNAtargeting mRNAs were determined by miRDB (www.mirdb.org), Targetscan (www.targetscan.org), and miRTarBase (mirtarbase.mbc.nctu.edu.tw). To further increase the reliability of the ceRNA network, intersecting lncRNAs and mRNAs in the miRNA predictions and TCGA-STAD differential expression data were selected. The ceRNA network interaction was visualized by Cytoscape v3.5.0 (28).

GC patients and tissue specimens

A total of 178 samples, consisting of paired GC tissues and normal gastric epithelial tissues, were obtained from GC patients that underwent radical surgery at Minhang Hospital Affiliated to Fudan University between 2011 and 2019. None of the patients received radiation therapy or chemotherapy prior to surgery. All cases were independently diagnosed by two experienced pathologists, and staging was determined according to the American Joint Committee on Cancer (AJCC) TNM classification (AJCC $7^{\text {th }}$ edition, 2010). Tissue specimens were immediately frozen in liquid nitrogen upon resection and stored at $-80^{\circ} \mathrm{C}$ for subsequent RNA extraction. Used human cancer tissues were approved by the Human Research Ethics Review Board of Minhang Hospital. Informed consent was also obtained from all patients.

GC cell lines and culture conditions

Nine human GC cell lines (BGC-823, AGS, MGC-803, HGC-27, MKN45, and SGC-7901), human umbilical vein endothelial cells (HUVECs) and human embryonic kidney (HEK) 293 FT cells were purchased from the Chinese Academy of Sciences (CAS) (Shanghai) Cell Bank. All cell lines were cultured in RPMI-1640 (Hyclone, 
Logan, UT, USA) supplemented with $10 \%$ fetal bovine serum at $37^{\circ} \mathrm{C}$ in a $5 \% \mathrm{CO}_{2}$ incubator.

\section{RNA extraction and qRT-PCR}

Total RNA was extracted from patient tissues and cell lines using TRIzol (Invitrogen, Carlsbad, CA, USA) according to the manufacturer's instructions. After, 1 $\mu \mathrm{g}$ of total RNA was used as template for the reverse transcription reaction to generate high-quality cDNA, using the Moloney Leukemia Virus Reverse Transcriptase Kit (Promega, Madison, Wisconsin, USA). cDNA samples were amplified by qRT-PCR using SYBR Green Mix (Promega) to measure lncRNA and mRNA expression, using GAPDH as a control for normalization. miRNA expression was measured by qRT-PCR using the All-in-One ${ }^{\mathrm{TM}}$ miRNA qRT-PCR kit (GeneCopoeia, Carlsbad, CA, USA) and ABI 7500 fast real-time PCR system (Applied Biosystems, Waltham, Massachusetts, USA), using U6 snRNA as an internal control. Each sample was measured three times and the fold change in relative expression was calculated by $2-\Delta \Delta \mathrm{CT}$. Primer sequences used in this study are shown in Table S1 of Supplementary Document 1.

\section{Immunohistochemistry (IHC)}

SERPINE1 expression in the collected tumor samples was determined by IHC staining. Tumor staining intensity was scored from 0 to 3 (0: No staining; 1: Weak staining; 2: Moderate staining; 3: Strong staining) based on the estimated percentage of tumor cells that are positively stained. To evaluate the mean staining intensity of tumor samples, at least 100 cells in multiple tumor areas were analyzed. Cytoplasmic expression was determined by Histoscore (H-score), using the formula: Histoscore $=$ $\sum(\mathrm{I} \times \mathrm{Pi})$, where $\mathrm{I}=$ Intensity, $\mathrm{Pi}=$ Percentage of positively stained tumor cells, and the score ranges from 0 to 300 (29). Scoring was performed by two independent pathologists, blinded to the clinical results. For animal studies, subcutaneous tumors were harvested from nude mice, paraffin-embedded, cut into $4 \mu \mathrm{m}$-thick sections, dewaxed and rehydrated. After antigen retrieval, tissue sections were incubated with 
anti-CD34 (CST) primary antibody at $4^{\circ} \mathrm{C}$ overnight, then incubated with anti-HRP secondary antibody (Dako, Glostrup, Denmark) for $2 \mathrm{~h}$ at room temperature and restained with Mayer's hematoxylin solution. IHC staining scoring was performed by two independent pathologists.

\section{Cell proliferation and colony formation assays}

To measure cell proliferation, tumor cells were seeded into 96-well plates at $24 \mathrm{~h}$ post-transfection and cultured for $7 \mathrm{~d}$. Cell counting kit-8 (CCK-8; Dojindo, Tokyo, Japan) solution $(10 \mu \mathrm{L})$ was added into each well daily. Cell proliferation was measured as the absorbance at $450 \mathrm{~nm}$. For colony formation, tumor cells were seeded into 6-well plates at 500 cells/well at $24 \mathrm{~h}$ post-transfection. After 2 weeks, cells were fixed in methanol and stained with $0.1 \%$ crystal violet. Colonies were counted using Quantity One (BioRad, Hercules, CA, USA). All experiments were repeated three times and the mean values were calculated.

Lentivirus production and cell transfection

Two types of lncNKX2-1-AS1-targeting lentiviral short-hairpin RNAs (shRNAs) were purchased from GenePharma (Shanghai, China) and transfected into GC cell lines. Lnc-NKX2-1-AS1-overexpressing plvx-IRES-zsGreen was purchased from Takara Bio (CA, USA). ShRNA and vector were transfected into the GC cell lines respectively. At $48 \mathrm{~h}$ post-transfection, cells were treated with $2 \mu \mathrm{g} / \mathrm{mL}$ puromycin for 2 weeks to select for cells with stable knockdown or overexpression of $\operatorname{lncNKX2-1-AS1.~Transfection~}$ efficiency was verified by qRT-PCR. Hsa-miR-145a-5p mimic, hsa-miR-145a-5p inhibitor and negative control (NC) oligonucleotides were purchased from Ribobio (Guangzhou, China). The plvx-IRES-zsGreen-SERPINE1 vector was purchased from Takara Bio (Catalog no. 632187, USA). GC cell lines were transfected with the above oligonucleotides and plasmids using Lipofectamine 3000 (Invitrogen), according to the manufacturer's instructions. 


\section{Cell migration and invasion assays}

Cell migration was examined using Transwell migration assay $(8-\mu \mathrm{m}$ pore size; Corning Costar, Cambridge, MA, USA). Non-adherent tumor cells, cultured in serumfree RPMI-1640 medium, were seeded into the upper chamber, and RPMI-1640 supplemented $20 \%$ serum was added into the lower chamber as a chemoattractant. After $24 \mathrm{~h}$ or $48 \mathrm{~h}$ of incubation, the inserted membranes were fixed in methanol and stained with $0.1 \%$ crystal violet. The upper surface of the membranes was gently wiped and cells migrating the bottom surface of the membrane were counted and visualized under the microscope. The cell invasion assay was performed similarly, with the addition of a layer of matrigel onto the Transwell membrane.

\section{Cell cycle and cell apoptosis}

For cell cycle analysis, cells were seeded into 6 -well plates at $3 \times 10^{5}$ cells/well and cultured at $37^{\circ} \mathrm{C}$ overnight. Adherent cells were collected and fixed in $70 \%$ cold ethanol at $-20^{\circ} \mathrm{C}$ overnight. Cells were stained with Propidium Iodide (PI) according to the manufacturer's instructions and cell cycle distribution was analyzed by flow cytometry. For cell apoptosis analysis, cells were cultured in $2 \mathrm{ml}$ culture medium in 6-well plates at $37^{\circ} \mathrm{C}$ for $18-24 \mathrm{~h}$, collected, washed twice in cold PBS, then resuspended in $100 \mu \mathrm{l}$ binding buffer (BD Pharmingen). Cells were stained with $5 \mu$ Annexin V-FITC and 5 $\mu 1$ PI solution for $20 \mathrm{~min}$ in the dark and then analyzed by flow cytometry within 30 min after staining. The apoptotic rate was calculated as follows: Apoptotic rate $=$ Early apoptotic (Q4) + late apoptotic (Q2). Cell cycle and apoptosis were measured by flow cytometry (FACS Calibur, BD Biosciences, San Jose, CA, USA).

\section{In vivo tumor formation}

Four-week-old female BALB/c nude mice were purchased from Shanghai SLAC Laboratory Animal Co., Ltd. and maintained under specific pathogen free conditions. AGS cells transfected with the NKX2-1-AS1 vector or the empty vector were subcutaneously injected into the right axillary of BALB/c nude mice. Tumor volume 
was measured once every 4 days to determine tumor formation. Mice were sacrificed 4 weeks post-injection and the xenograft tumors were harvested, weighed, and analyzed by qRT-PCR and IHC. All animal studies were approved by the Animal Care and Use Committee of Fudan University.

Fluorescence in situ hybridization (FISH)

AGS cells were fixed in 4\% PFA at room temperature for $15 \mathrm{~min}$, then permeabilized in $0.5 \%$ Triton $\mathrm{X}-100$ at $4^{\circ} \mathrm{C}$ for $15 \mathrm{~min}$. Cells were incubated with digoxigenin (DIG)-labeled NKX2-1-AS1 probes or Control-FISH probes at $55^{\circ} \mathrm{C}$ for 4 $\mathrm{h}$ then washed in 2X PBS for $5 \mathrm{~min}$. Horseradish peroxidase (HRP)-conjugated antiDIG secondary antibodies (Jackson, West Grove, PA, USA) were used for signal detection, and nuclei were counterstained with DAPI. Images were obtained using an Olympus confocal laser scanning microscope.

\section{Immunoblotting}

Total protein was extracted from cells using RIPA buffer (Cell Signaling Technology) and supplemented with protease and kinase inhibitors. Total cytoplasmic and nucleic proteins were extracted using the PROTTOT-1KT ProteoPrep kit (Sigma). Equal amounts $(30 \mu \mathrm{g})$ of protein from individual samples were separated by SDSPAGE and transferred onto a PVDF membrane. Membrane was blocked with 5\% BSA in PBS, then sequentially incubated with primary antibodies and the corresponding secondary antibodies. Proteins were visualized by Western Lightning Plus-ECL (PerkinElmer, Waltham, Massachusetts, USA). Antibodies: anti-SERPIN1(cat.11907, CST; 1:1000), anti-VEGFR-2(cat.9698, CST; 1:1000), anti-p-VEGFR-2(cat.2478, CST; 1:1000), anti-PLC- $\lambda$ (cat.5690, CST; 1:1000), anti-p-PLC- $\lambda$ (cat.8713, CST; 1:1000), anti-Erk1/2(cat.4695, CST; 1:1000), anti-p-Erk1/2(cat.4370, CST; 1:1000), antiP38(cat.8690, CST; 1:1000), anti-p-P38(cat.4511, CST; 1:1000), anti-FAK(cat.71433, CST; 1:1000), anti-p-FAK(cat.8556, CST; 1:1000), anti-Src(cat.2109, CST; 1:1000), anti-p-Src(cat.6943, CST; 1:1000), anti-Akt(cat.4685, CST; 1:1000), anti-p- 
Akt(cat.4060, CST; 1:1000), and anti-GAPDH(cat.51332, CST; 1:1000) primary antibodies, and HRP-conjugated goat anti-rabbit(cat.7074, CST; 1:3000) or anti-mouse IgG(cat.7076, CST; 1:3000) antibodies.

\section{Statistical analysis}

Statistical analysis was performed with SPSS 23.0 (SPSS, Chicago, IL, USA) or GraphPad Prism 7 (GraphPad Prism, Inc., La Jolla, CA, USA). Each experiment was repeated at least 3 times, and data are expressed as the mean $\pm \mathrm{SD}$ of three independent experiments. Two-group or three-group comparisons were performed using the Student's t-test or ANOVA, respectively. Association between NKX2-1-AS1 expression and clinicopathological variables was calculated using the chi-square test or Fisher's exact test. Survival curves were generated using the Kaplan-Meier estimator and difference between survival curves was analyzed using the rank sum test. $\mathrm{P}<0.05$ is considered statistically significant. 


\section{Results}

TCGA data analysis

lncRNA, miRNA, and mRNA differential expression analysis were performed on 343 GC samples and 30 adjacent non-tumor samples obtained from TCGA using the edgeR package in $\mathrm{R}$. After filtering by $\operatorname{logFC}>2$ and $\mathrm{P}<0.01,1,660$ DEmRNAs (910/54.8\% upregulated and 750/45.2\% downregulated), 102 miRNAs (85/81.0\% upregulated and 17/23.4\% downregulated) and $1034 \operatorname{lncRNAs}(819 / 79.2 \%$ upregulated and 215/20.8\% downregulated)were identified (Fig. 1A-F). A total of 137 DEmiRNAs were predicted using miRDB, Targetscan and miRTarBase and 471 target mRNAs were identified. Nine intersecting mRNAs that interact with 10 DEmiRNAs were selected between the 471 predicted target mRNAs and 1,622 DEmRNAs and used for the construction of the ceRNA network (Fig. 1G). To further elucidate the functions of these 1,622 DEmRNAs, KEGG pathway enrichment analysis was performed. In addition, 15 pathways associated with upregulated DEmRNAs and 28 pathways correlated with downregulated DEmRNAs were identified. In particular, the up- and downregulated DEmRNAs were most enriched in "Cell cycle" and "Neuroactive ligand-receptor interaction", respectively (Fig. 1H). To better understand the mechanisms by which lncRNAs bind to miRNAs and regulate mRNA expression in GC, a lncRNA-miRNAmRNA ceRNA network involving 62 DElncRNAs, 10 DEmiRNAS, and 9 DEmRNAs was constructed using Cytoscape 3.5.0 (Fig. 1I). Some mRNAs in the ceRNA network were previously reported to be associated with cancer, including Tribbles Pseudokinase 3, Serpin Family E Member 1 (SERPINE1), Mesoderm Specific Transcript (MEST), Kinesin Family Member 23 (KIF23), Transmembrane Protein 100 (TMEM100), and AAA Domain Containing 2 (ATAD2). Next, the association between the differentially expressed genes in the ceRNA network and the overall survival (OS) of GC patients was analyzed. We found that 9 of the 62 DElncRNAs and 2 of the 9 DEmRNAs, including NKX2-1-AS-1 and SERPINE1, were significantly associated with OS (Fig. $1 \mathrm{~J}, 1 \mathrm{~K}$, and data not shown). Given that the NKX2-1-AS1/miR145-5p/SERPINE1 was the only ceRNA regulatory axis that showed negative association with OS in both its 
lncRNA and mRNA ends, and that miR145-5p was expressed at low level in cancer tissues, our data suggest that the NKX2-1-AS1/miR145-5p/SERPINE1 axis plays a potential adverse role in the prognosis of GC patients.

NKX2-1-AS1 is overexpressed in GC- derived tumor tissues

NKX2-1-AS1 expression in 178 paired tumor/non-tumor tissue from GC patients was determined by qRT-PCR. Compared with the counterpart, GC tumor tissue showed upregulated NKX2-1-AS1 expression $(\mathrm{P}=0.0001)$, downregulated miR145-5p expression ( $\mathrm{P}=0.0033)$, and upregulated SERPINE1 expression $(\mathrm{P}=0.0004)$ (Fig. 2AC). Furthermore, clinical subgroup analysis also revealed that NKX2-1-AS1 expression was significantly upregulated in patients with advanced TNM staging (stage III-IV) and lymph node metastases ( $>6$ positive lymph nodes) (Fig. 2D, E). Although NKX2-1AS1 expression was positively correlated with larger tumor, infiltration of peritumoral tissues, peritoneum dissemination, and distant metastasis, there were no statistically significant differences among these subgroups (Additional file 2: Figure S1a-d). Overall, the clinical data show that NKX2-1-AS1 upregulation is often observed in GC and may therefore be associated with GC progression and metastasis.

NKX2-1-AS1 and SERPINE1 overexpression is positively correlated with GC progression and poor prognosis

To evaluate the prognostic value of NKX2-1-AS1 overexpression, the median score $(0.50)$ of relative NKX2-1-AS1 expression was defined as the cutoff value for dividing all GC patients into high- and low-expression groups (Additional file 2: Figure S1e). The correlation of NKX2-1-AS1 expression with the OS and progression-free survival (PFS) of GC patients was studied. Kaplan-Meier analysis showed that patients whose tumor tissue had high NKX-2-1AS1 expression exhibited significantly shorter 5 -year OS (40.52\% vs. 58.89\%, p = 0.0069) and 5-year PFS (36.67\% vs. 59.93\%, p = 0.0019), than patients with low NKX-2-1AS1 expression (Fig. 2F, 2G). Univariate 
analysis revealed that OS and PFS are correlated with T staging, N staging, TNM staging, distant metastasis, tumor tissue infiltration, peritoneal dissemination, and NKX2-1-AS1 expression in GC patients $(\mathrm{P}<0.01$, Table 1). Multivariate analysis indicated that NKX2-1-AS1 is an independent predictor for OS and PFS (Table 1). In addition, TNM staging, distant metastasis, and peripheral tumor tissue infiltration are independent risk factors for OS and PFS (Table 1). To determine the association between SERPINE1 expression and patient survival, GC patients were divided into low SERPINE1 expression $(0+$ and $1+; \mathrm{n}=89)$ or high SERPINE1 expression groups $(2+$ and $3+; \mathrm{n}=89$ ), based on SERPINE1 H-score in the corresponding tumor tissue samples. The median score $(0.50)$ of $\mathrm{H}$-score was defined as the cutoff value for dividing all GC patients into high- and low-expression groups (Additional file 2: Figure S1F). Compared with patients with low SERPINE1 expression, those with high SERPINE1 expression had shorter 5-year OS (39.48\% vs. 60.68\%, p = 0.0073). Altogether, these data suggest that NKX2-1-AS1 and SERPINE1 are potential prognostic predictors for GC patients.

NKX2-1-AS1 expression regulates GC cell proliferation, migration, and invasion

Given that NKX2-1-AS1 upregulation is positively associated with GC progression and metastasis, loss-of-function experiments were performed to confirm the effect of NKX2-1-AS1 on GC cell proliferation, migration, and invasion. AGS and HGC-27 cells, which have the highest and lowest NKX2-1-AS1 expression respectively, were used (Fig. 3A). shRNA against NKX2-1-AS1 was used to abrogate its expression, and assessed knockdown efficiency by qRT-PCR (Fig. 3B). Cell count and colony formation assays showed that NKX2-1-AS1 knockdown inhibited GC cell growth and colony formation (Fig. 3C, 3D), which could be reversed by overexpression of NKX21-AS1 (Fig. 4A-4C). Furthermore, NKX2-1-AS1 knockdown also suppressed the migratory and invasive features of GC cells (Fig. 3E, 3F), which were also reversed by NKX2-1-AS1 overexpression (Fig. 4D-E). Interestingly, there were no significant changes in cell cycle and apoptosis rate of GC cells after NKX2-1-AS1 knockdown 
(Fig. 3G, 3H) or overexpression (Fig. 4F, 4G). Collectively, these findings showed that NKX2-1-AS1 expression regulated GC cell growth, migration and invasion in vitro but had no impact on GC cell cycle and apoptosis.

NKX2-1-AS1 regulates GC cell proliferation and angiogenesis in vivo

To evaluate the effect of NKX2-1-AS1 on GC tissues in vivo, NKX2-1-AS1-stably expressing AGS cells were subcutaneously injected in female nude mice (Fig. 5A). As expected, xenograft tumors were larger and heavier in the AGS-NKX2-1-AS1 group than in the NC group (Fig. 5B, C). Similarly, NKX2-1-AS1 shRNA-transfected AGS was subcutaneously injected in female nude mice. In addition, it is observed that the tumors were smaller and lighter in the sh-NKX2-1-AS1 group when compared to the NC group (Fig. 5D, 5E). During necropsy, tumors appeared paler in the shNKX2-1AS1 group than in the NC group, and it is hypothesized that NKX2-1-AS1 expression might be associated with GC tissue angiogenesis, which in turn can impact tumor growth. IHC staining revealed enhanced CD34 expression in tumors overexpressing NKX2-1-AS1, which was reversed by NKX2-1-AS1 knockdown (Fig. 5F-H). Therefore, our in vivo results strongly suggest that NKX2-1-AS1 is positively correlated with GC tissue growth and increased angiogenesis.

\section{NKX2-1-AS1 is a ceRNA of miR-145-5p in GC}

To further elucidate the regulatory mechanism of NKX2-1-AS1 in GC, the subcellular localization of NKX2-1-AS1 was determined by FISH. As shown in Fig. 6A, the NKX2-1-AS1 transcript is primarily localized in the cytoplasm. Cytoplasmic lncRNAs are known to act as ceRNAs that attenuate negative regulation of target mRNAs by sponging miRNAs (30). Based on our TCGA data analysis and clinical sample validation, it is hypothesized that NKX2-1-AS1, miR-145-5p and SERPINE1 may be involved in a regulatory ceRNA network. It is predicted that the binding sites between NKX2-1-AS1 and miR-145-5p (Fig. 6B) and between miR-145-5p and SERPINE1 (Fig. 6C) using mircode and starbase, respectively. To validate our 
hypothesis, AGS and HGC-27 cells were transfected with miR-145-5p mimic or inhibitor (Fig. 6D) to up- and downregulate miR-145-5p expression, respectively. Knockdown of NKX2-1-AS1 significantly upregulated miR-145-5p. Conversely, down- and upregulation of miR-145-5p, respectively, increased and decreased NKX21-AS1 expression (Fig. 6E, 6F). Accordingly, NKX2-1-AS1 expression was negatively correlated with miR-145-5p expression in GC tissues (Fig. 6G). To obtain direct evidence of the interaction between NKX2-1-AS1 and miR-145-5p, NKX2-1-AS1 was subcloned with a wild-type (NKX2-1-AS1-WT) or mutated (NKX2-1-AS1-Mut) miR145-5p binding site into a dual-luciferase reporter. As shown in Fig. 6H, after cotransfection with miR-145-5p mimic, the relative luciferase activity of NKX2-1-AS1WT in HEK293FT cells was significantly decreased but the luciferase activity of NKX2-1-AS1-Mut was unchanged, demonstrating that miR-145-5p is a direct target of NKX2-1-AS1. Altogether, our data showed that NKX2-1-AS1 is a ceRNA that regulates $\mathrm{miR}-145-5 \mathrm{p}$.

NKX2-1-AS1 promotes GC tissue angiogenesis by targeting miR-145$5 \mathrm{p} / \mathrm{SERPINE} 1$

miRNA-mediated regulation of protein expression is accomplished through the inhibition of mRNA translation or degradation of mRNAs (31). According to our RNAseq analysis and binding site predictions from starbase, miR-145-5p binds to both SERPINE1 and MEST (Fig. 1I). Of these two upregulated mRNAs, SERPINE1 as its upregulation was negatively correlated with patient survival. Luciferase assay demonstrated that miR-145-5p binds complementary to the 3'-UTR of SERPINE1 (Fig. 7A). Next, whether SERPINE1 is also regulated by aberrant NKX2-1-AS1 expression is noted. It is observed that NKX2-1-AS1 knockdown or overexpression, respectively, downregulated or upregulated SERPINE1 mRNA expression (Fig. 7B). Consistently, miR-145-5p inhibitor could partially reverse the inhibitory effect of NKX2-1-AS1 on SERPINE1 expression (Fig. 7C). Likewise, AGS cells co-transfected with miR-145-5p mimic and plvx-SERPINE1 partially restored the expression of SERPINE1, compared 
with cells transfected with miR-145-5p mimic only (Fig. 7D). In our co-culture experiment, it is observed that HUVECs co-cultured with AGS display enhanced proliferation compared with HUVECs alone (Fig. 7E). In addition, HUVECs cocultured with AGS that overexpress both NKX2-1-AS1 and SERPINE were even more proliferative than those co-cultured with NKX2-1-AS1 knockdown AGS cells (Fig. 7F). Altogether, these findings demonstrate that the promoting effect of NKX2-1-AS1 in GC angiogenesis is primarily dependent on the miR-145-5p/SERPINE1 axis.

NKX2-1-AS1/ miR-145-5p/ SERPINE1 axis positively regulates GC metastasis, invasion, and angiogenesis via the VEGFR-2 signaling pathway

SERPINE1 was previously reported to play an important role in tissue angiogenesis. To determine whether NKX2-1-AS1/miR-145-5p regulates the canonical VEGFR2 signaling via SERPINE1, the expression and phosphorylation status of key proteins in the VEGFR2 signaling pathway were measured in GC cells following NKX2-1-AS1 overexpression or knockdown. Analyzed proteins include angiogenesisrelated VEGFR2, proliferation-related PLC $\gamma 1$ and ErK1/2, migration-related p38, FAK and Src, as well as survival-related Akt. It is observed that NKX2-1-AS1 knockdown and overexpression had no significant impact on total protein expression. In contrast NKX2-1-AS1 knockdown led to reduced protein phosphorylation, whereas NKX2-1AS1 overexpression resulted in increased protein phosphorylation, suggesting an active role of NKX2-1-AS1 in the regulation of VEGFR2 signaling. Similar results were obtained in HUVECS co-cultured with AGS cells (Fig. 8A). Overall, these findings indicated that regulation of GC cell metastasis by the NKX2-1-AS1/miR-145$5 \mathrm{p} / \mathrm{SERPINE} 1$ axis is dependent on the VEGFR2 pathway.

\section{Discussion}

Dysregulated lncRNA expression was recently found in GC and other gastrointestinal cancers (32). Previous studies have shown that NKX2-1-AS1 limits human carcinoma cell migration by negatively regulating CD274/PD-L1 and cell-cell 
interaction genes (12). Furthermore, its close proximity to the transcription factor NKX2-1 is known to contribute to GC progression (33). In this study, it is shown that NKX2-1-AS1 is significantly upregulated in GC, promoting cancer cell proliferation and supporting angiogenesis, both in vitro and in vivo. Furthermore, in vitro experiments showed a positive correlation of NKX2-A-AS1 with metastasis and invasion potential. Altogether, the results suggest that NKX2-1-AS1 promotes GC progression. Therefore, it is proposed that NKX2-1-AS1 can be used as a potential diagnostic and prognostic biomarker in the context of gastric cancer.

An increasing number of studies has demonstrated that the biological functions of lncRNAs are largely dependent on their unique subcellular localization (34). Cytoplasmic lncRNAs act as mRNA decoys, to regulate mRNA stability or translation, and influence signal transduction pathways (30). miR-145 was previously reported to inhibit GC cell migration and metastasis, likely due to the suppression of myosin VI (MYO6) expression (35). Using bioinformatic analysis and luciferase reporter assay, it is shown that NKX2-1-AS1 directly binds and inhibits miRNA-145-5p expression, and that miRNA-145-5p expression is significantly downregulated and negatively correlated with NKX2-1-AS1 expression in GC. Low miRNA-145-5p expression due to the ceRNA nature of NKX2-1-AS1, alleviates the downregulation of SERPINE1, thereby promoting GC progression. A similar ceRNA-related mechanism has been reported in nasopharyngeal cancer, in which MACC1 antisense RNA 1 (MACC1-AS1) acts as a molecular sponge of miR-145 (36). It was recently reported that, in cancer, miRNAs play a key role in signal transduction by degrading or inhibiting mRNAs (7).

It is well established that tumor angiogenesis is a key mechanism in promoting tumor growth and invasion (37). An increasing amount of evidence shows that miRNAs interact with several mRNA targets of the VEGF-A pathway and regulate GC-related angiogenesis (38). Conversely, miRNA-145 was shown to inhibit GC cell invasion, metastasis and angiogenesis by targeting v-ets erythroblastosis virus E26 oncogene homolog 1 (39). Additionally, inhibition of miRNA-mediated SERPINE1 limits tumor angiogenesis, regardless of the angiogenic stimuli, in malignant pleural mesothelioma 
(40). In the present study, it is found that SERPINE1 is a direct target of miR-145-5p. Further analysis revealed that the loss of SERPINE1 inhibition by miR-145-5p significantly enhanced VEGFR2 pathway activity. In contrast, the VEGFR2 pathway was significantly downregulated in GC cells overexpressing NKX2-1-AS1. Beyond SERPINE1, MEST mRNA, which exhibits potential miR-145-5p binding sites, was also significantly downregulated in GC samples obtained from TCGA database. However, MEST overexpression was not associated with GC patient prognosis and no differential expression was observed between tumor and non-tumor tissues in our samples (data not shown). Thus, it is hypothesized that miR-145-5p regulates SERPINE1 expression in GC. Co-culture of GC cells with HUVECs provided direct evidence of SERPINE1 in promoting vascular endothelial cell proliferation, further confirming its importance in angiogenesis. Taken together, our findings demonstrate that the NKX2-1-AS1/miR-145-5p axis activates the VEGFR2 signaling pathway by specifically upregulating SERPINE1 expression.

VEGFR2 is mainly expressed on the cell membrane and is found on both vascular endothelial and GC cells. Conversely, SERPINE1 proteins are mainly found in the cytoplasm and are directly associated with extracellular matrix remodeling. These findings suggest that SERPINE1 proteins are transported out of GC cells, such as by exosomes, and act on the tumor microenvironment to increase GC tissue angiogenesis, enhance GC cell proliferation and metastasis (Fig. 7B). This study highlighted the role of NKX2-1-AS1 as a ceRNA in the binding of miR-145-5p and upregulation of SERPINE1. This resulted in increased activation of the VEGFR2 signaling pathway and promoted tumor angiogenesis, proliferation and metastasis. The exact drivers of SERPINE1 localization change are still to uncover. To understand such mechanism and the molecular requirements of SERPINE1-VEGFR2 interaction, will be key to better clarify the biological processes implicated in GC progression.

\section{Conclusion}

In summary, this study showed that NKX2-1-AS1 upregulation is frequent and 
might be an independent prognostic biomarker in GC. NKX2-1-AS1 is a lncRNA that promotes in vitro and in vivo GC-cell proliferation and tumor angiogenesis. Our findings elucidate the molecular mechanisms by which NKX2-1-AS1 directly targets miR-145-5p to activate VEGFR2 signaling and consequently promote GC progression (Fig. 8B). Therefore, it is proposed that NKX2-1-AS1 might be a new prognostic biomarker and a potential therapeutic target in the context of gastric cancer. 
Abbreviations

GC: Gastric cancer; lncRNAs : Long non-coding RNAs; TCGA: The Cancer Genome Atlas; ceRNA: competing endogenous RNA; FISH: fluorescence in situ hybridization; SERPINE1: serpin family E member 1; NKX2-1 antisense RNA 1: NKX2-1-AS1; miRNAs: microRNAs; uPA: urokinase-type plasminogen activator; UPAR: cellular receptor for urokinase-type plasminogen activator; PAI-1: plasminogen activator inhibitor-1; HUVECs: human umbilical vein endothelial cells; STAD: Stomach adenocarcinoma; FC: fold change; FDR: false discovery rate; AJCC: American Joint Committee on Cancer; HEK: human embryonic kidney; IHC: Immunohistochemistry; H-score: Histoscore; shRNAs: short-hairpin RNAs; NC: negative control; PI: Propidium Iodide; DIG: digoxigenin; HRP: Horseradish peroxidase; MEST: Mesoderm Specific Transcript; KIF23: Kinesin Family Member 23; TMEM100: Transmembrane Protein 100; ATAD2: AAA Domain Containing 2; PFS: progression-free survival; MACC1-AS1: MACC1 antisense RNA 1.

Ethics approval and consent to participate

All aspects of this study were approved by the Human Research Ethics Review Board of Minhang Hospital University of Fudan University. Written informed consent was obtained from all enrolled patients, and all relevant investigations were performed according to the principles of the Declaration of Helsinki. All animal studies were performed with approval from the Institutional Animal Care and Use Committee of Fudan University.

Consent for publication

Consent to publish has been obtained from all authors.

Availability of data and materials

All data in our study are available upon request. 


\section{Competing interests}

The authors declare that they have no competing interests.

\section{Funding}

This study was supported by grants from the Shanghai Minhang District Natural Science Research Project Foundation (Grant Nos. 2019MHZ091); Fundamental Medical Project of Minhang Hospital of Fudan University Project Foundation (Grant Nos. 2019MHJC04).

\section{Authors' contributions}

FT and JXZ contributed equally to this study; FT, YC, XDS, YJG and PHW performed all experiments; ML, SQL and YOC collected tissue samples and the clinical data; JXZ analyzed and interpreted the data; FT, JXZ, and SQL drafted the manuscript. All authors read and approved the final manuscript.

\section{Acknowledgements}

Not applicable. 


\section{References}

1. Torre LA, Bray F, Siegel RL, Ferlay J, Lortet-Tieulent J, Jemal A. Global cancer statistics, 2012. CA: a cancer journal for clinicians. 2015;65(2):87-108. Epub 2015/02/06. doi: 10.3322/caac.21262. PubMed PMID: 25651787.

2. Van Cutsem E, Sagaert X, Topal B, Haustermans K, Prenen H. Gastric cancer. Lancet (London, England). 2016;388(10060):2654-64. Epub 2016/05/10. doi: 10.1016/s0140-6736(16)30354-3. PubMed PMID: 27156933.

3. Catalano V, Labianca R, Beretta GD, Gatta G, de Braud F, Van Cutsem E. Gastric cancer. Critical reviews in oncology/hematology. 2009;71(2):127-64. Epub 2009/02/24. doi: 10.1016/j.critrevonc.2009.01.004. PubMed PMID: 19230702.

4. Deng M, Zeng C, Lu X, He X, Zhang R, Qiu Q, et al. miR-218 suppresses gastric cancer cell cycle progression through the CDK6/Cyclin D1/E2F1 axis in a feedback loop. Cancer letters. 2017;403:175-85. Epub 2017/06/22. doi: 10.1016/j.canlet.2017.06.006. PubMed PMID: 28634044. 5. Nienhüser H, Schmidt T. Angiogenesis and Anti-Angiogenic Therapy in Gastric Cancer. International journal of molecular sciences. 2017;19(1). Epub 2018/01/04. doi: 10.3390/ijms19010043. PubMed PMID: 29295534; PubMed Central PMCID: PMCPMC5795993.

6. Chen M, Wu X, Ma W, Zhou Q, Wang X, Zhang R, et al. Decreased expression of IncRNA VPS9D1-AS1 in gastric cancer and its clinical significance. Cancer biomarkers : section A of Disease markers. 2017;21(1):23-8. Epub 2017/10/19. doi: 10.3233/cbm-170172. PubMed PMID: 29036784. 7. Bracken CP, Scott HS, Goodall GJ. A network-biology perspective of microRNA function and dysfunction in cancer. Nature reviews Genetics. 2016;17(12):719-32. Epub 2016/11/01. doi: 10.1038/nrg.2016.134. PubMed PMID: 27795564.

8. Xue X, Yang YA, Zhang A, Fong KW, Kim J, Song B, et al. LncRNA HOTAIR enhances ER signaling and confers tamoxifen resistance in breast cancer. Oncogene. 2016;35(21):2746-55. Epub 2015/09/15. doi: 10.1038/onc.2015.340. PubMed PMID: 26364613; PubMed Central PMCID: PMCPMC4791209.

9. Kong J, Sun W, Li C, Wan L, Wang S, Wu Y, et al. Long non-coding RNA LINC01133 inhibits epithelial-mesenchymal transition and metastasis in colorectal cancer by interacting with SRSF6. Cancer letters. 2016;380(2):476-84. Epub 2016/07/23. doi: 10.1016/j.canlet.2016.07.015. PubMed PMID: 27443606.

10. Li Z, Wu X, Gu L, Shen Q, Luo W, Deng C, et al. Long non-coding RNA ATB promotes malignancy of esophageal squamous cell carcinoma by regulating miR-200b/Kindlin-2 axis. Cell death \& disease. 2017;8(6):e2888. Epub 2017/06/24. doi: 10.1038/cddis.2017.245. PubMed PMID: 28640252; PubMed Central PMCID: PMCPMC5520904.

11. Ma L, Zhou Y, Luo X, Gao H, Deng X, Jiang Y. Long non-coding RNA XIST promotes cell growth and invasion through regulating miR-497/MACC1 axis in gastric cancer. Oncotarget. 2017;8(3):4125-35. Epub 2016/12/03. doi: 10.18632/oncotarget.13670. PubMed PMID: 27911852; PubMed Central PMCID: PMCPMC5354817.

12. Kathuria H, Millien G, McNally L, Gower AC, Tagne JB, Cao Y, et al. NKX2-1-AS1 negatively regulates CD274/PD-L1, cell-cell interaction genes, and limits human lung carcinoma cell migration. Scientific reports. 2018;8(1):14418. Epub 2018/09/28. doi: 10.1038/s41598-018-327935. PubMed PMID: 30258080; PubMed Central PMCID: PMCPMC6158174.

13. Wang J, Ding $Y, W u Y$, Wang $X$. Identification of the complex regulatory relationships related to gastric cancer from IncRNA-miRNA-mRNA network. Journal of cellular biochemistry. 
2020;121(1):876-87. Epub 2019/08/28. doi: 10.1002/jcb.29332. PubMed PMID: 31452262.

14. Rakic JM, Maillard C, Jost M, Bajou K, Masson V, Devy L, et al. Role of plasminogen activatorplasmin system in tumor angiogenesis. Cellular and molecular life sciences : CMLS. 2003;60(3):463 73. Epub 2003/05/10. doi: 10.1007/s000180300039. PubMed PMID: 12737307.

15. Binder BR, Mihaly J, Prager GW. UPAR-UPA-PAI-1 interactions and signaling: a vascular biologist's view. Thrombosis and haemostasis. 2007;97(3):336-42. Epub 2007/03/06. PubMed PMID: 17334498.

16. Yang JD, Ma L, Zhu Z. SERPINE1 as a cancer-promoting gene in gastric adenocarcinoma: facilitates tumour cell proliferation, migration, and invasion by regulating EMT. Journal of chemotherapy (Florence, Italy). 2019;31(7-8):408-18. Epub 2019/11/15. doi: 10.1080/1120009x.2019.1687996. PubMed PMID: 31724495.

17. Liao P, Li W, Liu R, Teer JK, Xu B, Zhang W, et al. Genome-scale analysis identifies SERPINE1 and SPARC as diagnostic and prognostic biomarkers in gastric cancer. OncoTargets and therapy. 2018;11:6969-80. Epub 2018/11/10. doi: 10.2147/ott.s173934. PubMed PMID: 30410354; PubMed Central PMCID: PMCPMC6199229.

18. Bajou K, Noël A, Gerard RD, Masson V, Brunner N, Holst-Hansen C, et al. Absence of host plasminogen activator inhibitor 1 prevents cancer invasion and vascularization. Nature medicine. 1998:4(8):923-8. Epub 1998/08/13. doi: 10.1038/nm0898-923. PubMed PMID: 9701244.

19. Gutierrez LS, Schulman A, Brito-Robinson T, Noria F, Ploplis VA, Castellino FJ. Tumor development is retarded in mice lacking the gene for urokinase-type plasminogen activator or its inhibitor, plasminogen activator inhibitor-1. Cancer research. 2000;60(20):5839-47. Epub 2000/11/04. PubMed PMID: 11059781.

20. Bajou K, Maillard C, Jost M, Lijnen RH, Gils A, Declerck P, et al. Host-derived plasminogen activator inhibitor-1 (PAI-1) concentration is critical for in vivo tumoral angiogenesis and growth. Oncogene. 2004;23(41):6986-90. Epub 2004/08/03. doi: 10.1038/sj.onc.1207859. PubMed PMID: 15286708.

21. Bajou K, Masson V, Gerard RD, Schmitt PM, Albert V, Praus M, et al. The plasminogen activator inhibitor PAl-1 controls in vivo tumor vascularization by interaction with proteases, not vitronectin. Implications for antiangiogenic strategies. The Journal of cell biology. 2001;152(4):777-84. Epub 2001/03/27. doi: 10.1083/jcb.152.4.777. PubMed PMID: 11266468; PubMed Central PMCID: PMCPMC2195770.

22. Waltz DA, Natkin LR, Fujita RM, Wei Y, Chapman HA. Plasmin and plasminogen activator inhibitor type 1 promote cellular motility by regulating the interaction between the urokinase receptor and vitronectin. The Journal of clinical investigation. 1997;100(1):58-67. Epub 1997/07/01. doi: 10.1172/jci119521. PubMed PMID: 9202057; PubMed Central PMCID: PMCPMC508165.

23. Loskutoff DJ, Curriden SA, Hu G, Deng G. Regulation of cell adhesion by PAI-1. APMIS : acta pathologica, microbiologica, et immunologica Scandinavica. 1999;107(1):54-61. Epub 1999/04/06. doi: 10.1111/j.1699-0463.1999.tb01526.x. PubMed PMID: 10190280.

24. Bajou K, Peng H, Laug WE, Maillard C, Noel A, Foidart JM, et al. Plasminogen activator inhibitor-1 protects endothelial cells from FasL-mediated apoptosis. Cancer cell. 2008;14(4):324 34. Epub 2008/10/07. doi: 10.1016/j.ccr.2008.08.012. PubMed PMID: 18835034; PubMed Central PMCID: PMCPMC2630529.

25. Masuda T, Hattori N, Senoo T, Akita S, Ishikawa N, Fujitaka K, et al. SK-216, an inhibitor of plasminogen activator inhibitor-1, limits tumor progression and angiogenesis. Molecular cancer 
therapeutics. 2013;12(11):2378-88. Epub 2013/08/31. doi: 10.1158/1535-7163.mct-13-0041. PubMed PMID: 23990114.

26. Robinson MD, McCarthy DJ, Smyth GK. edgeR: a Bioconductor package for differential expression analysis of digital gene expression data. Bioinformatics (Oxford, England). 2010;26(1):139-40. Epub 2009/11/17. doi: 10.1093/bioinformatics/btp616. PubMed PMID: 19910308; PubMed Central PMCID: PMCPMC2796818.

27. Ito K, Murphy D. Application of ggplot2 to Pharmacometric Graphics. CPT: pharmacometrics \& systems pharmacology. 2013;2(10):e79. Epub 2013/10/18. doi: 10.1038/psp.2013.56. PubMed PMID: 24132163; PubMed Central PMCID: PMCPMC3817376.

28. Shannon P, Markiel A, Ozier O, Baliga NS, Wang JT, Ramage D, et al. Cytoscape: a software environment for integrated models of biomolecular interaction networks. Genome research. 2003;13(11):2498-504. Epub 2003/11/05. doi: 10.1101/gr.1239303. PubMed PMID: 14597658; PubMed Central PMCID: PMCPMC403769.

29. Yeo W, Chan SL, Mo FK, Chu CM, Hui JW, Tong JH, et al. Phase I/II study of temsirolimus for patients with unresectable Hepatocellular Carcinoma (HCC) - a correlative study to explore potential biomarkers for response. BMC cancer. 2015;15:395. Epub 2015/05/13. doi: 10.1186/s12885-015-1334-6. PubMed PMID: 25962426; PubMed Central PMCID: PMCPMC4434865.

30. Schmitt AM, Chang HY. Long Noncoding RNAs in Cancer Pathways. Cancer cell. 2016;29(4):452-63. Epub 2016/04/14. doi: 10.1016/j.ccell.2016.03.010. PubMed PMID: 27070700; PubMed Central PMCID: PMCPMC4831138.

31. Sandler A, Gray R, Perry MC, Brahmer J, Schiller JH, Dowlati A, et al. Paclitaxel-carboplatin alone or with bevacizumab for non-small-cell lung cancer. The New England journal of medicine. 2006;355(24):2542-50. Epub 2006/12/15. doi: 10.1056/NEJMoa061884. PubMed PMID: 17167137. 32. Yang XZ, Cheng TT, He QJ, Lei ZY, Chi J, Tang Z, et al. LINC01133 as ceRNA inhibits gastric cancer progression by sponging miR-106a-3p to regulate APC expression and the $\mathrm{Wnt} / \beta$-catenin pathway. Molecular cancer. 2018;17(1):126. Epub 2018/08/24. doi: 10.1186/s12943-018-0874-1. PubMed PMID: 30134915; PubMed Central PMCID: PMCPMC6106894.

33. Zhao BW, Jiang SS, Chen YM, Huang CY, Li YF. Reduced NKX2.1 expression predicts poor prognosis of gastric carcinoma. PloS one. 2014;9(12):e114556. Epub 2014/12/06. doi: 10.1371/journal.pone.0114556. PubMed PMID: 25478793; PubMed Central PMCID: PMCPMC4257675.

34. Chen LL. Linking Long Noncoding RNA Localization and Function. Trends in biochemical sciences. 2016;41(9):761-72. Epub 2016/08/09. doi: 10.1016/j.tibs.2016.07.003. PubMed PMID: 27499234.

35. Lei C, Du F, Sun L, Li T, Li T, Min Y, et al. miR-143 and miR-145 inhibit gastric cancer cell migration and metastasis by suppressing MYO6. Cell death \& disease. 2017;8(10):e3101. Epub 2017/10/13. doi: 10.1038/cddis.2017.493. PubMed PMID: 29022908; PubMed Central PMCID: PMCPMC5682659.

36. Chen $\mathrm{S}$, Luo $\mathrm{X}, \mathrm{Wu} \mathrm{W}, \mathrm{Li} \mathrm{Y}, \mathrm{Yu} \mathrm{H}$, Wang $\mathrm{Y}$, et al. The long non-coding RNA MACC1-AS1 promotes nasopharyngeal carcinoma cell stemness via suppressing miR-145-mediated inhibition on SMAD2/MACC1-AS1 axis. Biomedicine \& pharmacotherapy $=$ Biomedecine \& pharmacotherapie. 2020;125:109986. Epub 2020/02/15. doi: 10.1016/j.biopha.2020.109986. PubMed PMID: 32058221. 
37. Sammarco G, Varricchi G, Ferraro V, Ammendola M, De Fazio M, Altomare DF, et al. Mast Cells, Angiogenesis and Lymphangiogenesis in Human Gastric Cancer. International journal of molecular sciences. 2019;20(9). Epub 2019/05/01. doi: 10.3390/ijms20092106. PubMed PMID: 31035644; PubMed Central PMCID: PMCPMC6540185.

38. Zhang X, Tang J, Zhi X, Xie K, Wang W, Li Z, et al. Correction: miR-874 functions as a tumor suppressor by inhibiting angiogenesis through STAT3/VEGF-A pathway in gastric cancer. Oncotarget. 2017;8(17):29535. Epub 2017/05/05. doi: 10.18632/oncotarget.17402. PubMed PMID: 28468128; PubMed Central PMCID: PMCPMC5438749.

39. Zheng L, Pu J, Qi T, Qi M, Li D, Xiang X, et al. miRNA-145 targets v-ets erythroblastosis virus E26 oncogene homolog 1 to suppress the invasion, metastasis, and angiogenesis of gastric cancer cells. Molecular cancer research : MCR. 2013;11(2):182-93. Epub 2012/12/13. doi: 10.1158/15417786.mcr-12-0534. PubMed PMID: 23233482.

40. Takayama Y, Hattori N, Hamada H, Masuda T, Omori K, Akita S, et al. Inhibition of PAI-1 Limits Tumor Angiogenesis Regardless of Angiogenic Stimuli in Malignant Pleural Mesothelioma. Cancer research. 2016;76(11):3285-94. Epub 2016/05/20. doi: 10.1158/0008-5472.can-15-1796. PubMed PMID: 27197170. 
Figure legend

Fig.1 RNA-seq data analysis of gastric adenocarcinoma in TCGA database. A-C Clustered heat maps of the differentially expressed RNAs in gastric cancer tissues and adjacent non-tumor gastric tissues. Rows represent RNAs, whereas columns represent gastric cancer tissues and adjacent non-tumor gastric tissue samples. Differentially expressed lncRNAs, miRNAs, and mRNAs in gastric cancer tissues and adjacent non-tumor gastric tissue samples. $\log 2 \mathrm{FC}>1.5$ and $\mathrm{FDR}<0.05$. FC: folds change; FDR: false discovery rate. D-F Volcano plots were used to visualize and assess the variation of (D) long non-coding RNAs, (E) microRNAs, and (F) mRNAs expression between gastric cancer tissues and adjacent non-tumor gastric tissues. The values of the $\mathrm{x}$ - and y-axes indicate the averaged normalized signal values of the group (log scaled). $\mathbf{G}$ Identification of 471 commonly changed targeted mRNAs of 137 DEmiRNAs from the three publicly profile datasets (miRDB, Targetscan and miRTarBase). The cross areas meant the number of commonly changed mRNAs between DEmRNAs and target mRNAs is 9, which includes SERPINE1. H All enriched KEGG pathways for differentially expressed mRNAs (the bar plot shows the enrichment scores of the significantly enriched KEGG pathways). KEGG, Kyoto Encyclopedia of Genes and Genomes. I The lncRNA-miRNA-mRNA ceRNA network. Blue squares, downregulated miRNAs; blue circles, downregulated mRNAs; blue diamonds, downregulated lncRNAs. Red squares, upregulated miRNAs; red circles, upregulated mRNAs; red diamonds, upregulated lncRNAs. J-K Kaplan-Meier survival curves for NKX2-1-AS1 and SERPINE1 associated with overall survival. Horizontal axis: overall survival time (years); vertical axis: survival function.

Fig.2 NKX2-1-AS1 and SERPINE1 overexpression is positively correlated with GC progression and poor prognosis. A-C Relative expression of NKX2-1-AS1, miR145-5p and SERPINE1 detected by qRT-PCR in 178 paired GC cancer tissues and matched normal tissues. Results are presented as $\Delta$ cycle threshold $(\Delta \mathrm{Ct})$ in tumor tissues relative to normal tissues. D-E Relative expression of NKX2-1-AS1 in GC with different TNM stage and different numbers of positive regional lymph nodes. F-G 
Kaplan-Meier plots of the OS and PFS of GC patients with high $(\mathrm{n}=89)$ and low $(\mathrm{n}=$ 89) levels of NKX2-1-AS1. Data are presented as the mean \pm SD. H Kaplan-Meier plots of the OS of GC patients with high $(\mathrm{n}=89)$ and low $(\mathrm{n}=89)$ levels of SERPINE1. Data are presented as the mean \pm SD. I IHC staining of SERPINE1 in patients with gastric cancer (GC); a, tumor tissue without positive staining (IHC 0+); b, tumor tissue with a partial weak staining (IHC 1+); c, tumor tissue with weak to moderate staining (IHC 2+); d, tumor tissue with strong staining (IHC 3+).

Fig.3 Reduced expression of NKX2-1-AS1 decreases proliferation, migration and invasion in GC cells. A qRT-PCR analysis of the relative expression of NKX2-1-AS1 in six GC cell lines. B qRT-PCR was conducted to verify the relative expression of NKX2-1-AS1 in AGS and HGC-27 cells transfected with two independent shRNAs targeting NKX2-1-AS1. C CCK-8 assay of AGS and HGC-27 cells after knockdown of NKX2-1-AS1. D-H Representative results of the colony formation, transwell, cell cycle, and apoptosis assays of AGS and HGC-27 cells after shNKX2-1-AS1-1 or shNKX2-1-AS1-2 transfection.

Fig.4 Overexpression of NKX2-1-AS1 promotes proliferation, migration and invasion in GC cells. A Relative expression of NKX2-1-AS1 confirmed by qRT-PCR in AGS and HGC-27 cells with NKX2-1-AS1 overexpression. B-E Proliferation, migration, and invasion assays of AGS and HGC-27 cells with NKX2-1-AS1 overexpression by the CCK-8 assay and transwell assay. F-G Representative results of the cell cycle and apoptosis assays of AGS and HGC-27 cells after NKX2-1-AS1 overexpression.

Fig.5 NKX2-1-AS1 regulates GC cell proliferation and angiogenesis in vivo. A The right armpit was injected with AGS cells transfected with NKX2-1-AS1 expression vector or empty vector and shNKX2-1-AS1-1 or shNKX2-1-AS1-NC in upper panel. Representative images of xenograft tumors are indicated in the bottom panel. B-E 
Tumor volume and weight of the xenograft in NKX2-1-AS1 overexpression groups and control group or NKX2-1-AS1 knowdown group and control group. F Representative IHC staining results of CD34 in corresponding xenografts. G-H Statistical analysis of $\mathrm{H}$-score of CD34 in corresponding xenografts. Error bars: mean $\pm \mathrm{SD}$ from three independent experiments.

Fig.6 NKX2-1-AS1 is a ceRNA of miR-145-5p in GC. A FISH detection for NKX21-AS1 (red) was performed in AGS cells. The nucleus was counterstained with DAPI (blue). B Schematic representation of the predicted binding site for miR-145-5p in NKX2-1-AS1 by online database Mircode Predicted algorithm. The numbers indicate the positions of the nucleotides in the reference wild-type sequence of NKX2-1-AS1 (Ensembl version: ENSG00000253563). C Schematic representation of the predicted miR-145-5p target site within the 3'-UTR of SERPINE1. The predicted target site for miR-145-5p is located at the proximal portion of the SERPINE1 3'-UTR. Two nucleotides complementary to the seed sequence of miR-145-5p were mutated in the SERPINE1 mutant plasmid. The number indicates the position of the nucleotides in the reference wild-type sequence of SERPINE1 (NM_000602.5). D, E Relative expression of miR-145-5p in AGS and HGC-27 cells transfected with miR-145-5p mimic or inhibitor c or cells after transfection with shNKX2-1-AS1 or scramble sequence (E). F Relative expression of NKX2-1-AS1 in AGS and HGC-27 cells transfected with miR145-5p mimic or inhibitor. G Correlation analysis between NKX2-1-AS1 and miR-145$5 \mathrm{p}$ expression in $178 \mathrm{GC}$ tumor tissues. H Relative luciferase activities of wild type (WT) and mutated (Mut) NKX2-1-AS1 reporter plasmid in human embryonic kidney (HEK) 293FT cells co-transfected with miR-145-5p mimic. Error bars: mean \pm SD. n.s, not significant, $* \mathrm{P}<0.05, * * \mathrm{P}<0.01$ and $* * * \mathrm{P}<0.001$

Fig.7 NKX2-1-AS1 promotes GC tissue angiogenesis by targeting miR-1455p/SERPINE1. A Luciferase reporter assay in HEK-293FT cells co-transfected with wide type (WT) or mutated (Mut) SERPINE1 3'-UTR reporter vector and miR-145-5p 
mimic. B Relative expression of SERPINE1 mRNA in AGS cells with NKX2-1-AS1 knockdown or HGC-27 cells with NKX2-1-AS1 overexpression. C qRT-PCR was conducted to evaluate the mRNA expression of SERPINE1 gene in AGS cells following reduced expression of NKX2-1 -AS1 and/or inhibition of miR-145-5p. D qRT-PCR was performed to access the mRNA expression of SERPINE1 gene in AGS cells following the ectopic expression of miR-145-5p and/or plvx-APC expression vector lacking the 3'-UTR. E The CCK-8 experiment was used to detect the cell proliferation activity of HUVECs cells when cultured alone and co-cultured with AGS cells. F NKX2-1-AS1 knocked down or overexpressed or SERPINE1 overexpressed AGS cells were cocultured with HUVECs cells, CCK-8 experiment was used to detect HUVECs cell proliferation activity.

Fig.8 NKX2-1-AS1/ miR-145-5p/ SERPINE1 axis positively regulates GC metastasis, invasion, and angiogenesis via the VEGFR-2 signaling pathway. A Immunoblot assay of SERPIN1, VEGFR-2, p-VEGFR-2, PLC- $\lambda$, p-PLC- $\lambda$, Erk1/2, pErk1/2, P38, p-P38, FAK, p-FAK, Src, p-Src, Akt, and p-Akt proteins in AGS cells transfected with shNKX2-1-AS1-1 and/or NKX2-1-AS1. Numbers showed quantification of relative protein amount. GAPDH was used as an internal control. After HUVECs cells were co-cultured with corresponding AGS cells for 7 days, the content of VEGFR-2 and p-VEGFR-2 protein was detected. GAPDH serves as an internal reference. B Schematic diagram of the regulatory mechanism of NKX2-1-AS1/miR145-5p/SERPINE1 axis in the promotion of GC proliferation, metastasis and angiogenesis.

Additional file 1: Table S1. Primers and oligonucleotides sequences used in this study.

Additional file 2: Figure S1. The expression of NKX2-1-AS1 in GC patents with different clinical subgroups. A-D Relative expression of NKX2-1-AS1 in GC with different tumor sizes, with/without infiltration of peritumoral tissues (IPT), 
with/without peritoneum dissemination (PD), and with/without distant metastasis. Results were presented as $\Delta$ cycle threshold $(\Delta \mathrm{Ct})$ in tumor tissues relative to normal tissues. $\mathbf{E}$ qRT-PCR was performed to examine NKX2-1-AS1 expression in $178 \mathrm{GC}$ cancer tissues. Relative expression of NKX2-1-AS1 was presented as log2 (fold change of $\Delta \mathrm{Ct}$ value) in tumor tissue to that of matched normal tissues. GC patients were divided into high $(\mathrm{n}=89)$ and low $(\mathrm{n}=89)$ groups according to the median value $(0.50)$. F H-score of SERPINE1 expression in $178 \mathrm{GC}$ cancer tissues. GC patients were divided into high $(\mathrm{n}=89)$ and low $(\mathrm{n}=89)$ groups according to the median value $(0.50)$. 
Figures
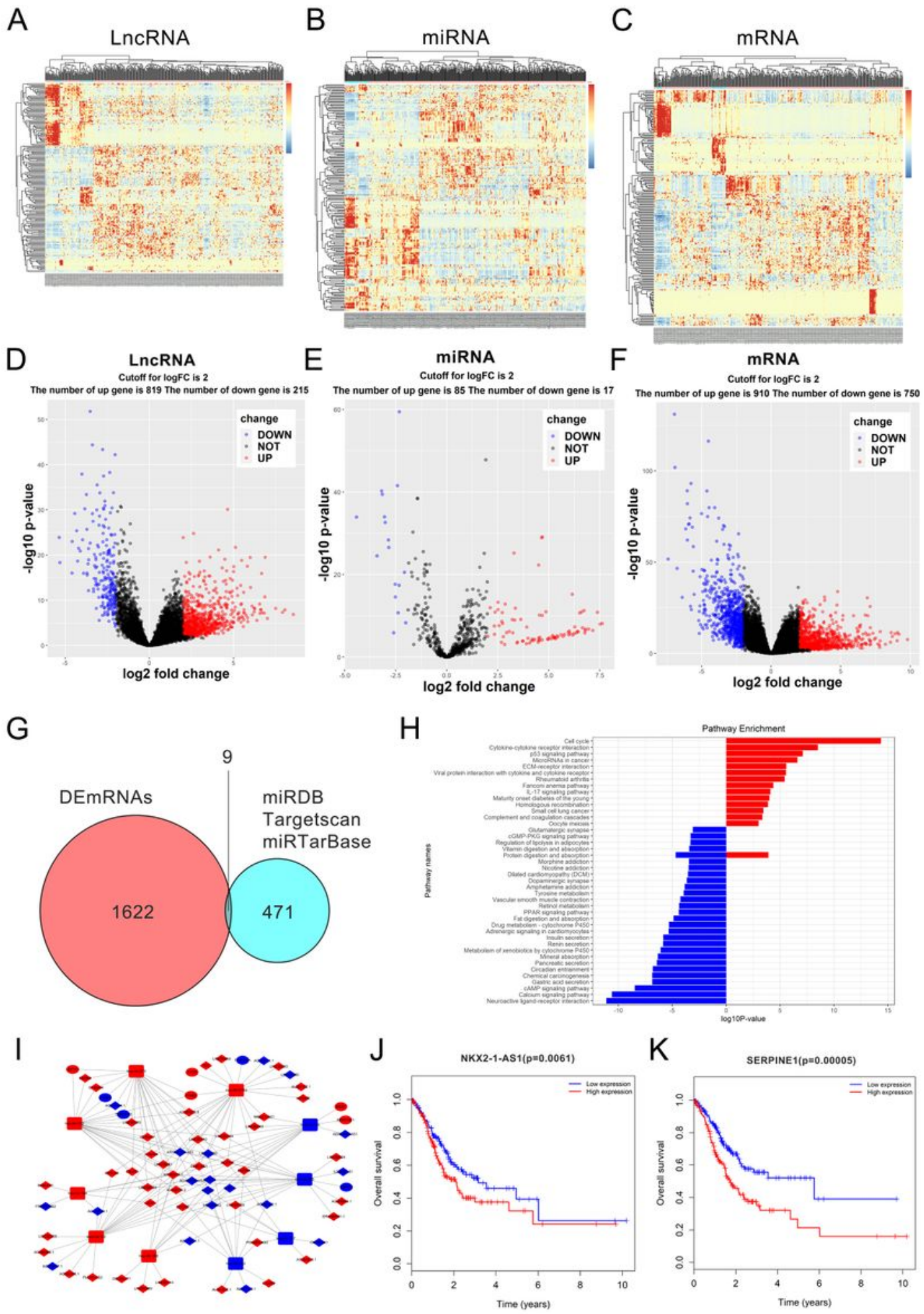

\section{Figure 1}

RNA-seq data analysis of gastric adenocarcinoma in TCGA database. A-C Clustered heat maps of the differentially expressed RNAs in gastric cancer tissues and adjacent non囚tumor gastric tissues. Rows represent RNAs, whereas columns represent gastric cancer tissues and adjacent non\tumor gastric tissue 
samples. Differentially expressed IncRNAs, miRNAs, and mRNAs in gastric cancer tissues and adjacent non邓tumor gastric tissue samples. log2FC $>1.5$ and FDR<0.05. FC: folds change; FDR: false discovery rate. D-F Volcano plots were used to visualize and assess the variation of (D) long non $₫$ coding RNAs, (E) microRNAs, and (F) mRNAs expression between gastric cancer tissues and adjacent non囚tumor gastric tissues. The values of the $x \bigotimes$ and $y \llbracket$ axes indicate the averaged normalized signal values of the group (log scaled). G Identification of 471 commonly changed targeted mRNAs of 137 DEmiRNAs from the three publicly profile datasets (miRDB, Targetscan and miRTarBase). The cross areas meant the number of commonly changed mRNAs between DEmRNAs and target mRNAs is 9, which includes SERPINE1. H All enriched KEGG pathways for differentially expressed mRNAs (the bar plot shows the enrichment scores of the significantly enriched KEGG pathways). KEGG, Kyoto Encyclopedia of Genes and Genomes. I The IncRNAØmiRNA囚mRNA ceRNA network. Blue squares, downregulated miRNAs; blue circles, downregulated mRNAs; blue diamonds, downregulated IncRNAs. Red squares, upregulated miRNAs; red circles, upregulated mRNAs; red diamonds, upregulated IncRNAs. J-K Kaplan囚Meier survival curves for NKX2-1AS1 and SERPINE1 associated with overall survival. Horizontal axis: overall survival time (years); vertical axis: survival function. 
A

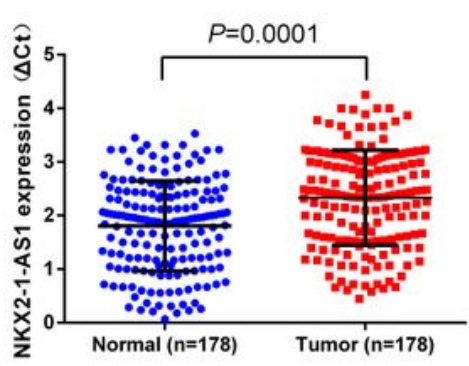

C

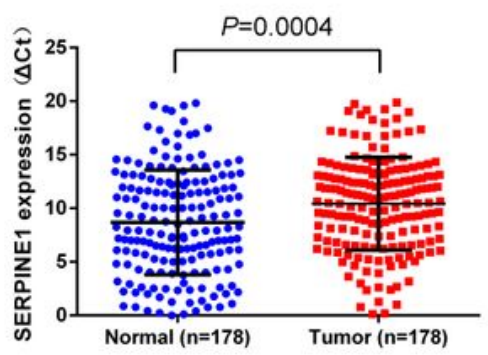

E

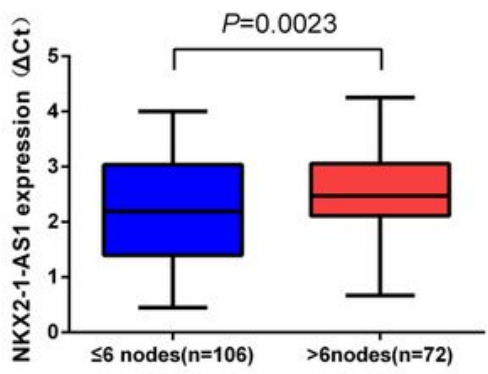

G

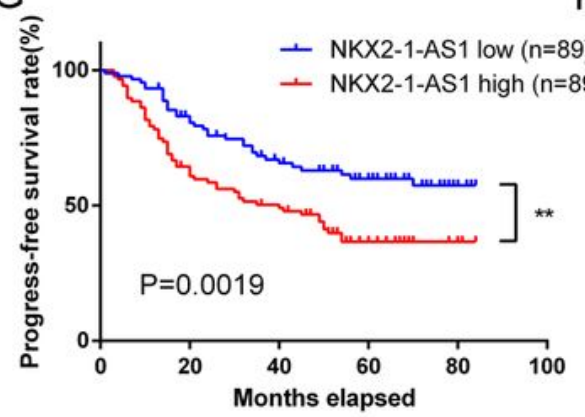

I

SERPINE1 $(0+) \quad$ SERPINE1 $(1+)$

B

D

$\mathrm{F}$

$\mathrm{H}$
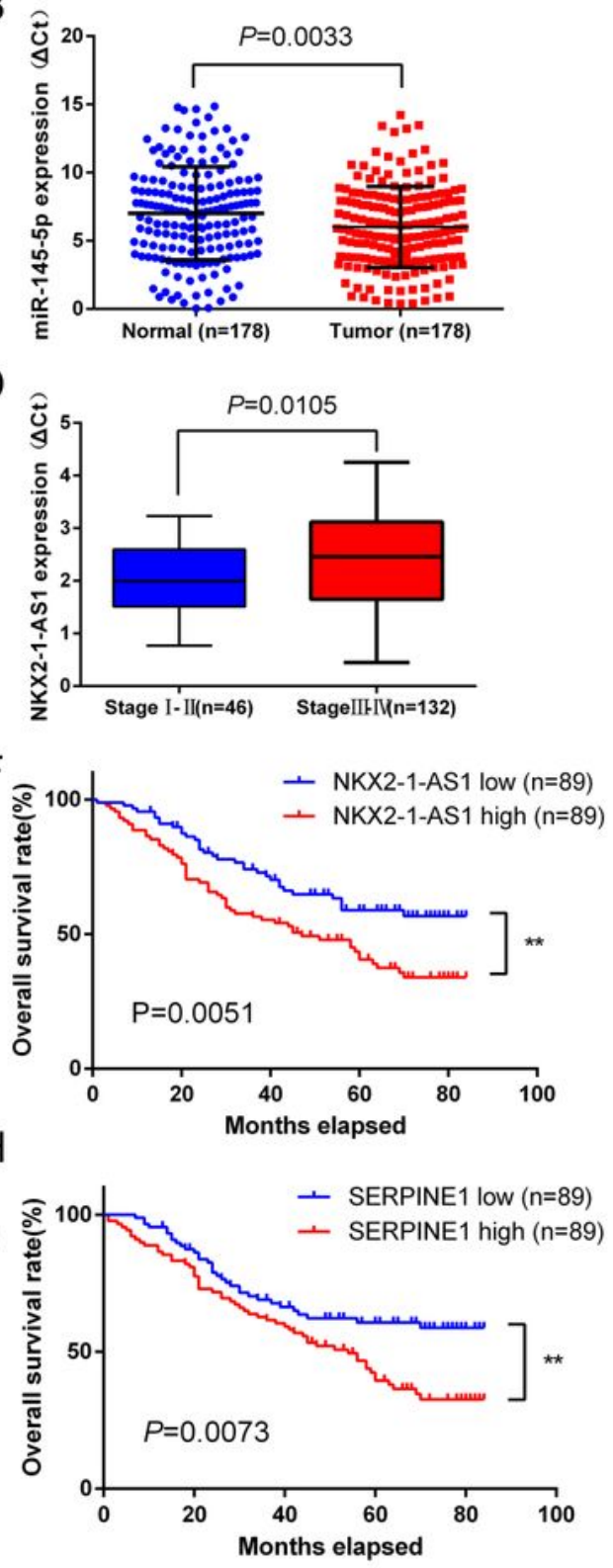

SERPINE1 $(2+)$

SERPINE1 $(3+)$

gastric

cancer

tissue
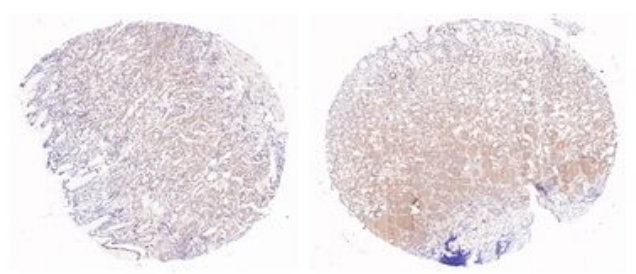

\section{Figure 2}

NKX2-1-AS1 and SERPINE1 overexpression is positively correlated with GC progression and poor prognosis. A-C Relative expression of NKX2-1-AS1, miR-145-5p and SERPINE1 detected by qRT-PCR in 178 paired GC cancer tissues and matched normal tissues. Results are presented as $\Delta$ cycle threshold $(\Delta \mathrm{Ct})$ in tumor tissues relative to normal tissues. D-E Relative expression of NKX2-1-AS1 in GC with different TNM stage and different numbers of positive regional lymph nodes. F-G 28 Kaplan-Meier plots 
of the OS and PFS of GC patients with high $(n=89)$ and low $(n=89)$ levels of NKX2-1-AS1. Data are presented as the mean \pm SD. H Kaplan-Meier plots of the OS of GC patients with high $(n=89)$ and low $(n$ $=89$ ) levels of SERPINE1. Data are presented as the mean \pm SD. I IHC staining of SERPINE1 in patients with gastric cancer (GC); a, tumor tissue without positive staining (IHC $0+$ ); $b$, tumor tissue with a partial weak staining $(\mathrm{IHC} 1+)$; c, tumor tissue with weak to moderate staining $(\mathrm{IHC} 2+)$; d, tumor tissue with strong staining ( $\mathrm{IHC} 3+)$.
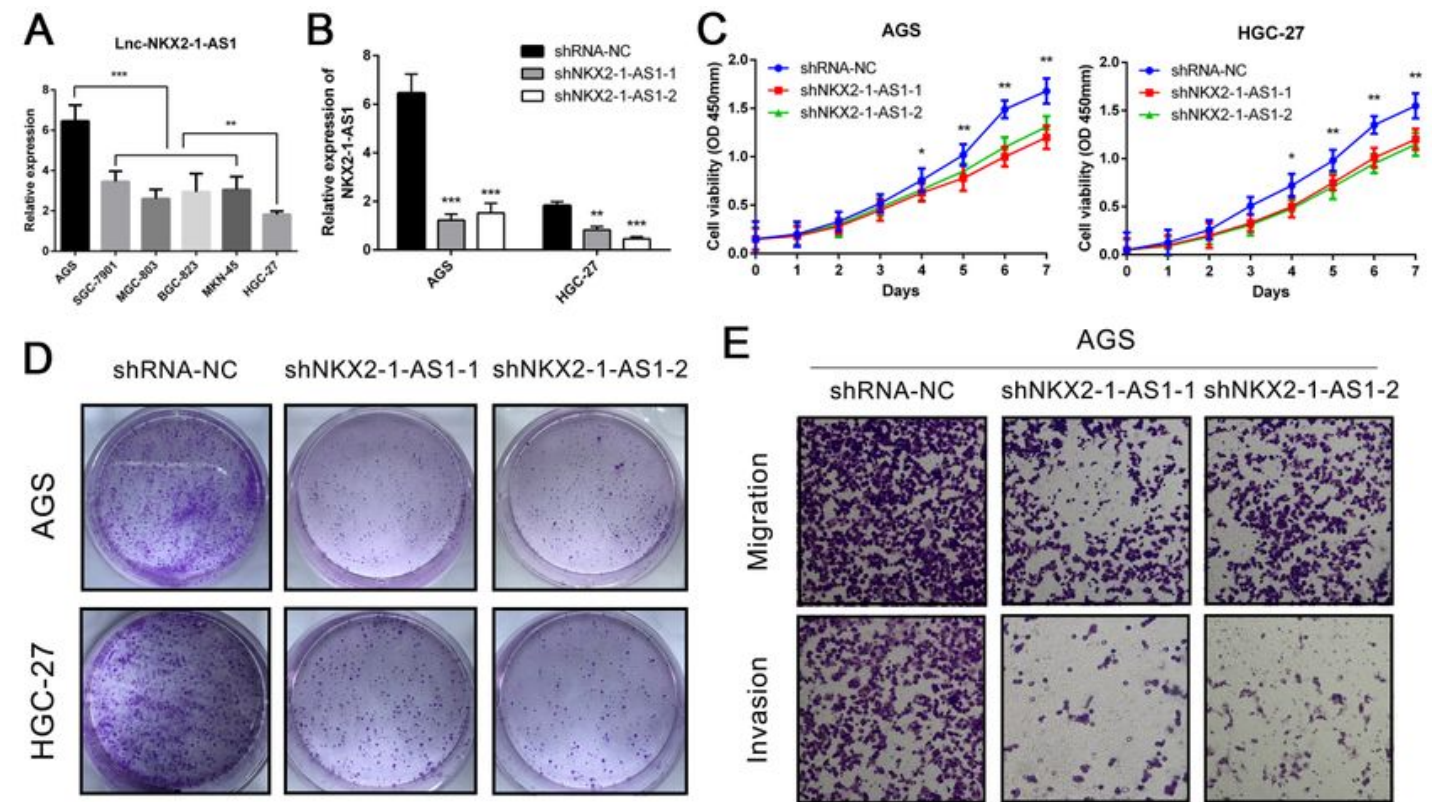

$\mathrm{E}$

AGS
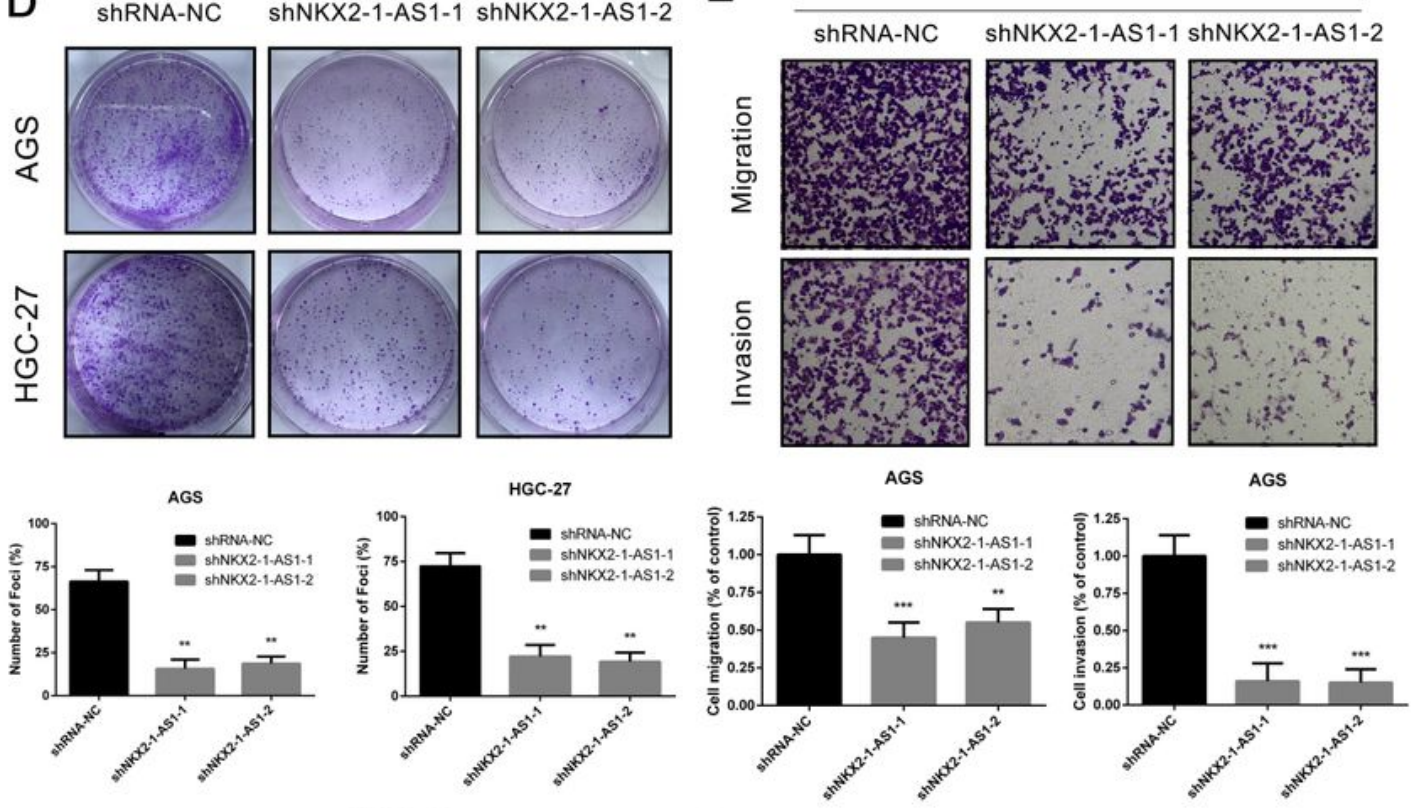

$\mathrm{F}$

HGC-27

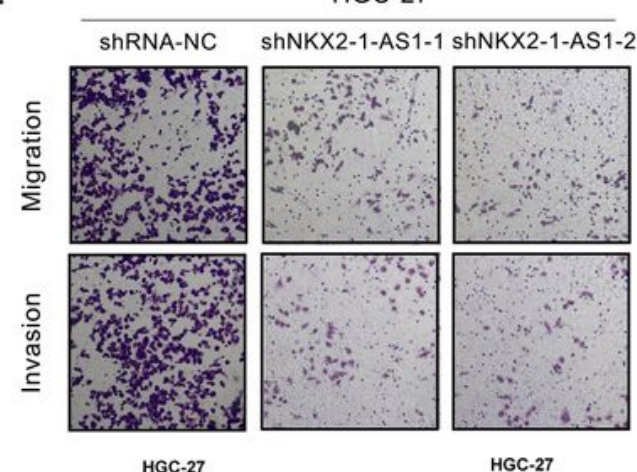

G
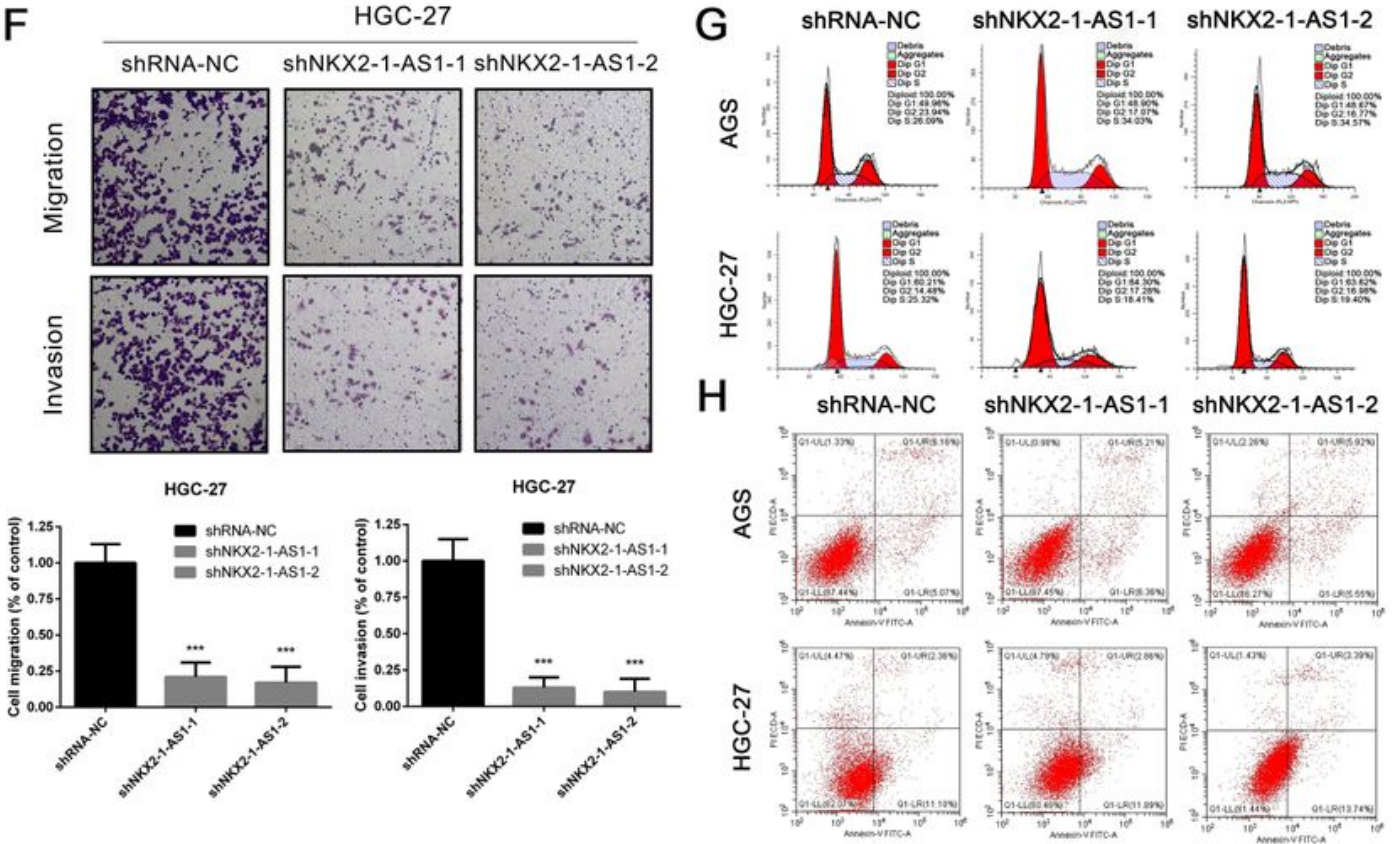

Figure 3 
Reduced expression of NKX2-1-AS1 decreases proliferation, migration and invasion in GC cells. A qRTPCR analysis of the relative expression of NKX2-1-AS1 in six GC cell lines. B qRT-PCR was conducted to verify the relative expression of NKX2-1-AS1 in AGS and HGC-27 cells transfected with two independent shRNAs targeting NKX2-1-AS1. C CCK-8 assay of AGS and HGC-27 cells after knockdown of NKX2-1-AS1. D-H Representative results of the colony formation, transwell, cell cycle, and apoptosis assays of AGS and HGC-27 cells after shNKX2-1-AS1-1 or shNKX2-1-AS1-2 transfection.

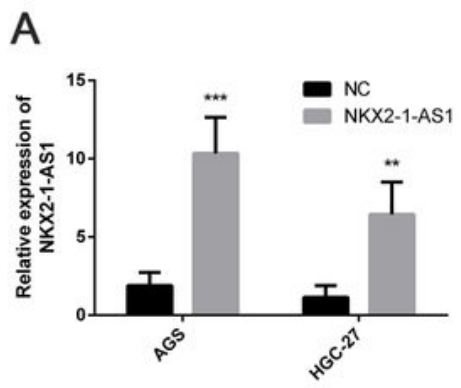

D
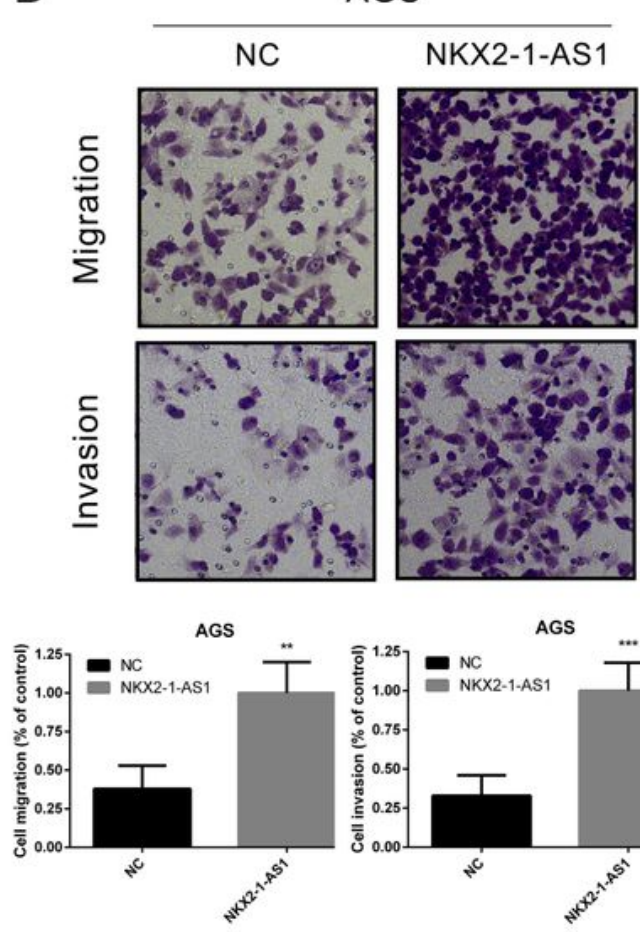

$\mathrm{F}$

NC
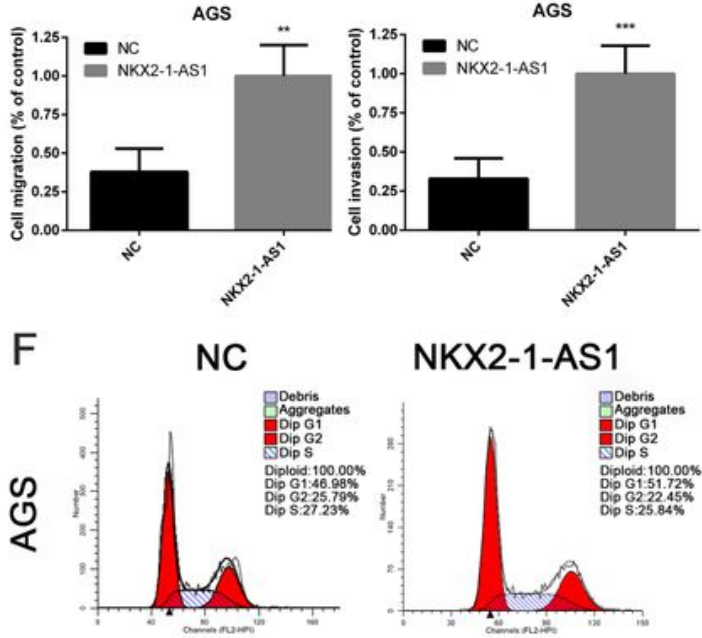

NKX2-1-AS1
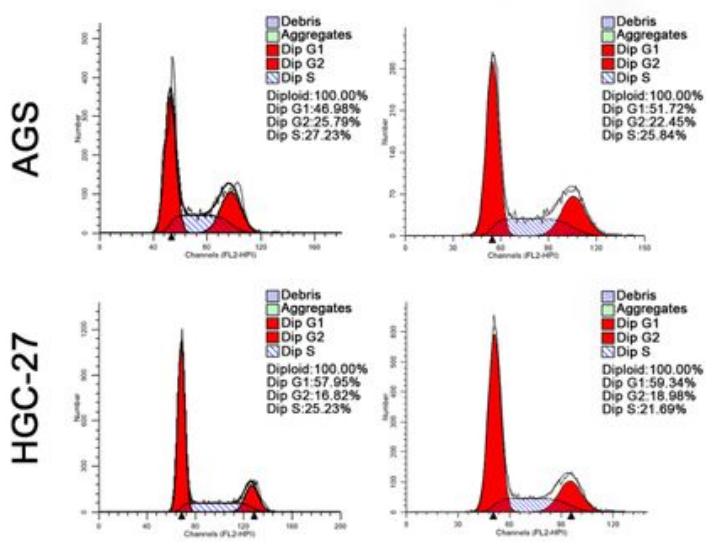

AGS

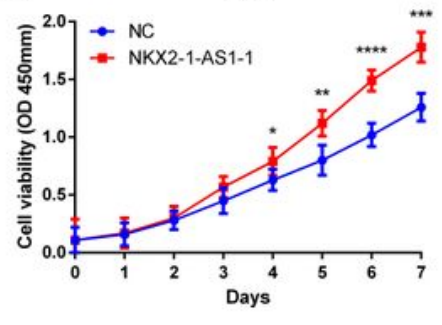

E

C

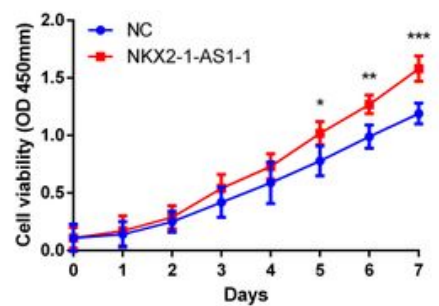

HGC-27
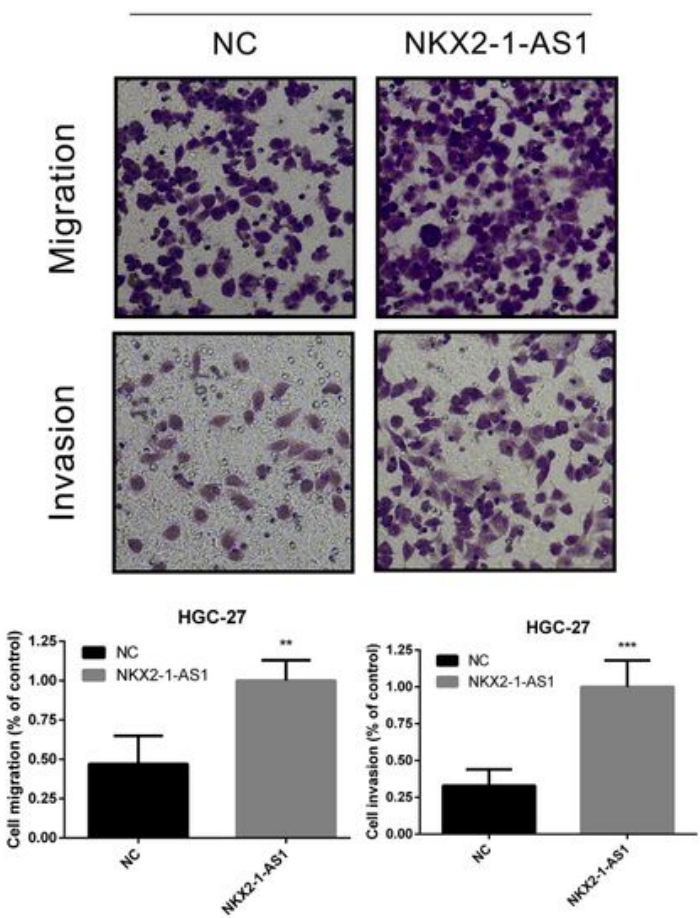

G

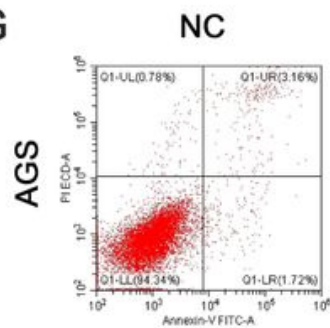

NKX2-1-AS1
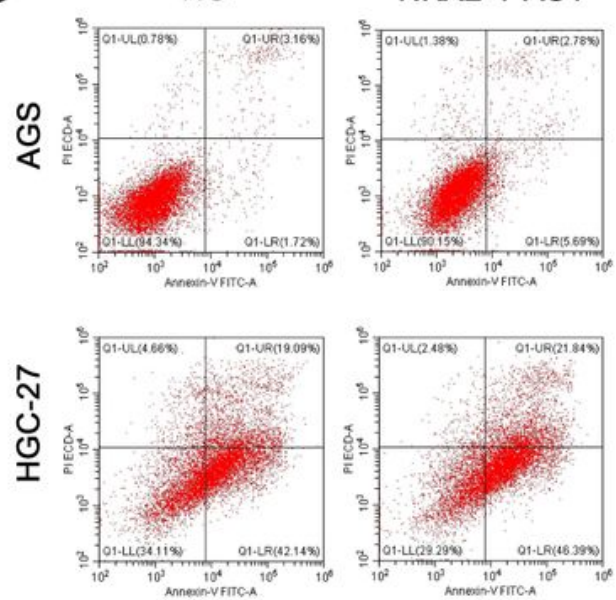
Overexpression of NKX2-1-AS1 promotes proliferation, migration and invasion in GC cells. A Relative expression of NKX2-1-AS1 confirmed by qRT-PCR in AGS and HGC-27 cells with NKX2-1-AS1 overexpression. B-E Proliferation, migration, and invasion assays of AGS and HGC-27 cells with NKX2-1AS1 overexpression by the CCK-8 assay and transwell assay. F-G Representative results of the cell cycle and apoptosis assays of AGS and HGC-27 cells after NKX2-1-AS1 overexpression.
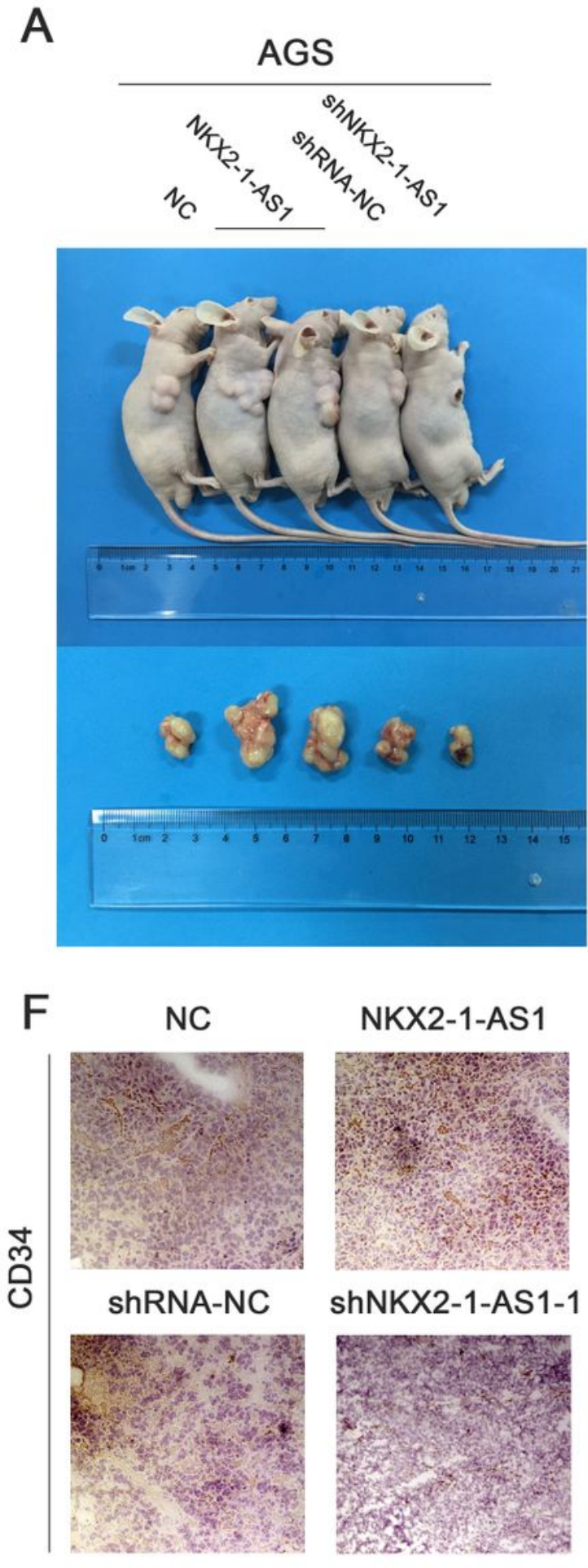

B

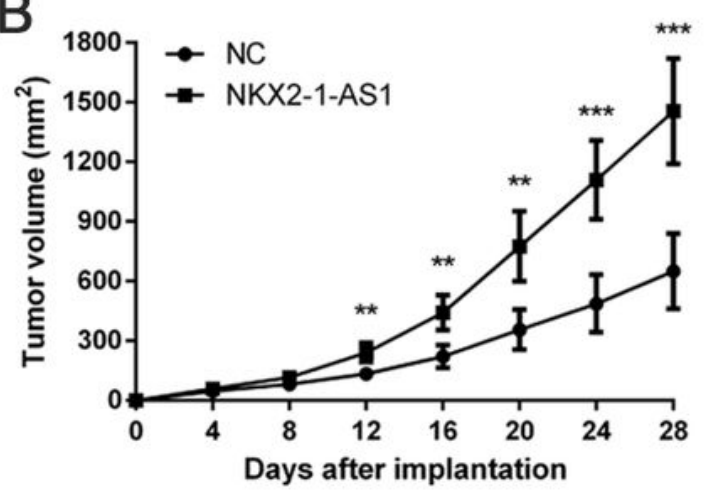

C

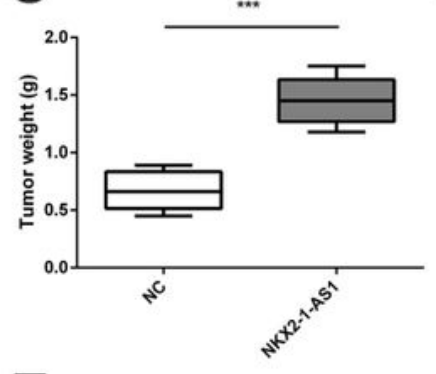

E

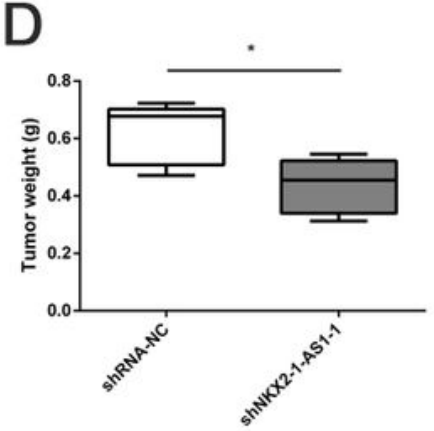

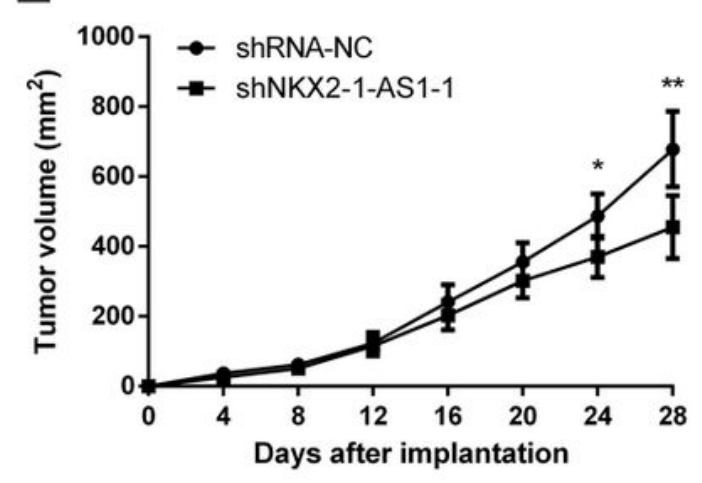

G
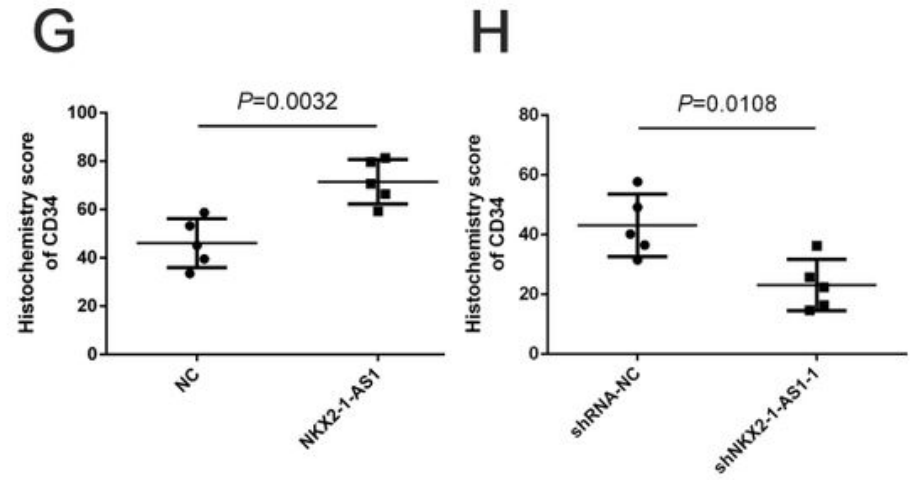

Figure 5 
NKX2-1-AS1 regulates GC cell proliferation and angiogenesis in vivo. A The right armpit was injected with AGS cells transfected with NKX2-1-AS1 expression vector or empty vector and shNKX2-1-AS1-1 or shNKX2-1-AS1-NC in upper panel. Representative images of xenograft tumors are indicated in the bottom panel. B-E 29 Tumor volume and weight of the xenograft in NKX2-1-AS1 overexpression groups and control group or NKX2-1-AS1 knowdown group and control group. F Representative IHC staining results of CD34 in corresponding xenografts. G-H Statistical analysis of H-score of CD34 in corresponding xenografts. Error bars: mean \pm SD from three independent experiments.
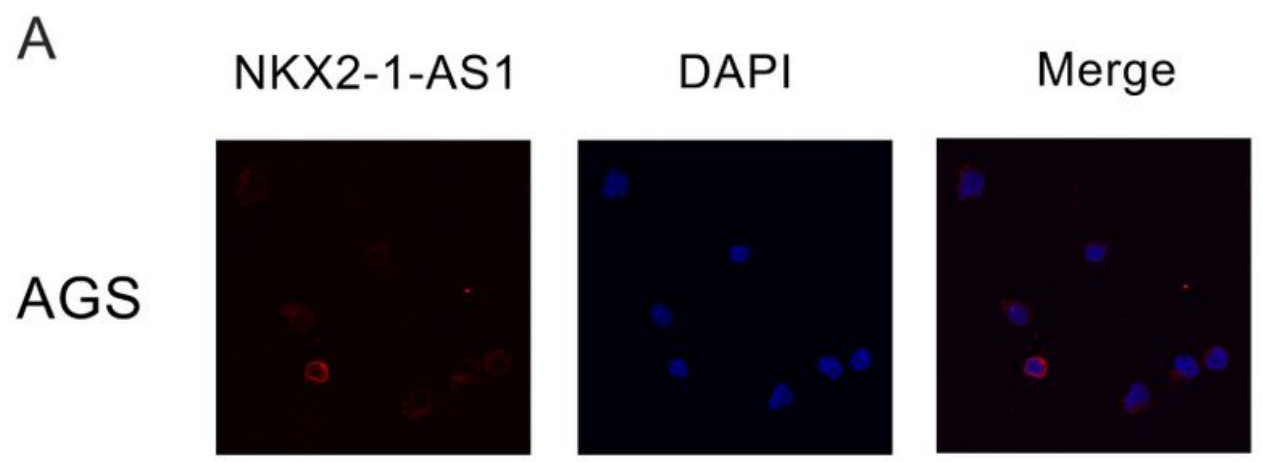

B

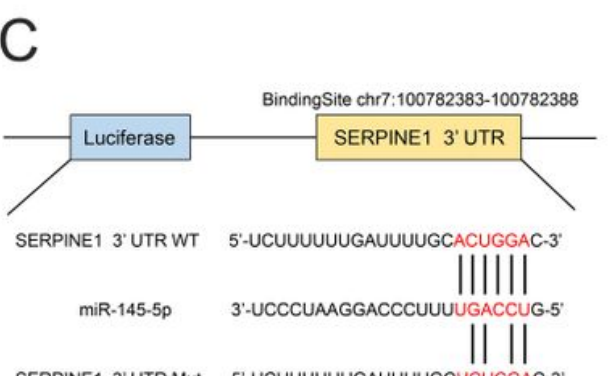

SERPINE1 3' UTR MUt $\quad 5^{\prime}$-UCUUUUUUGAUUUUGCUCUCGAC-3'
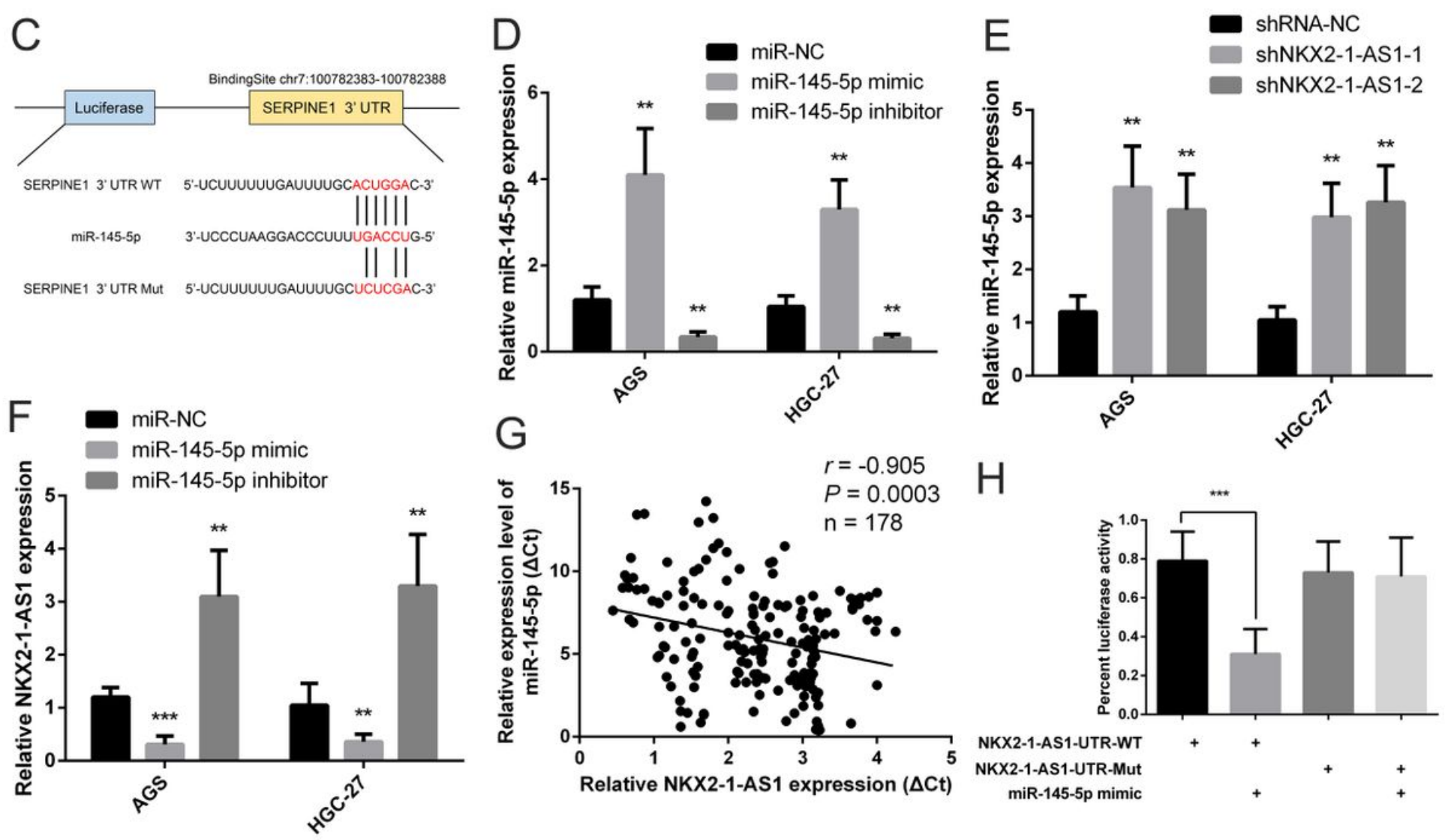

NKX2-1-AS1 AGCUGAACUGGAGUGG

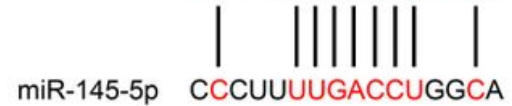

Chr14:36991745-36991760

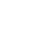


version: ENSG00000253563). C Schematic representation of the predicted miR-145-5p target site within the 3'-UTR of SERPINE1. The predicted target site for miR-145-5p is located at the proximal portion of the SERPINE1 3'-UTR. Two nucleotides complementary to the seed sequence of miR-145-5p were mutated in the SERPINE1 mutant plasmid. The number indicates the position of the nucleotides in the reference wildtype sequence of SERPINE1 (NM_000602.5). D, E Relative expression of miR-145-5p in AGS and HGC-27 cells transfected with miR-145-5p mimic or inhibitor $c$ or cells after transfection with shNKX2-1-AS1 or scramble sequence (E). F Relative expression of NKX2-1-AS1 in AGS and HGC-27 cells transfected with miR-145-5p mimic or inhibitor. G Correlation analysis between NKX2-1-AS1 and miR-145-5p expression in $178 \mathrm{GC}$ tumor tissues. H Relative luciferase activities of wild type (WT) and mutated (Mut) NKX2-1-AS1 reporter plasmid in human embryonic kidney (HEK) 293FT cells co-transfected with miR-145-5p mimic. Error bars: mean \pm SD. n.s, not significant, $* P<0.05, * * P<0.01$ and ${ }^{* * * P}<0.001$
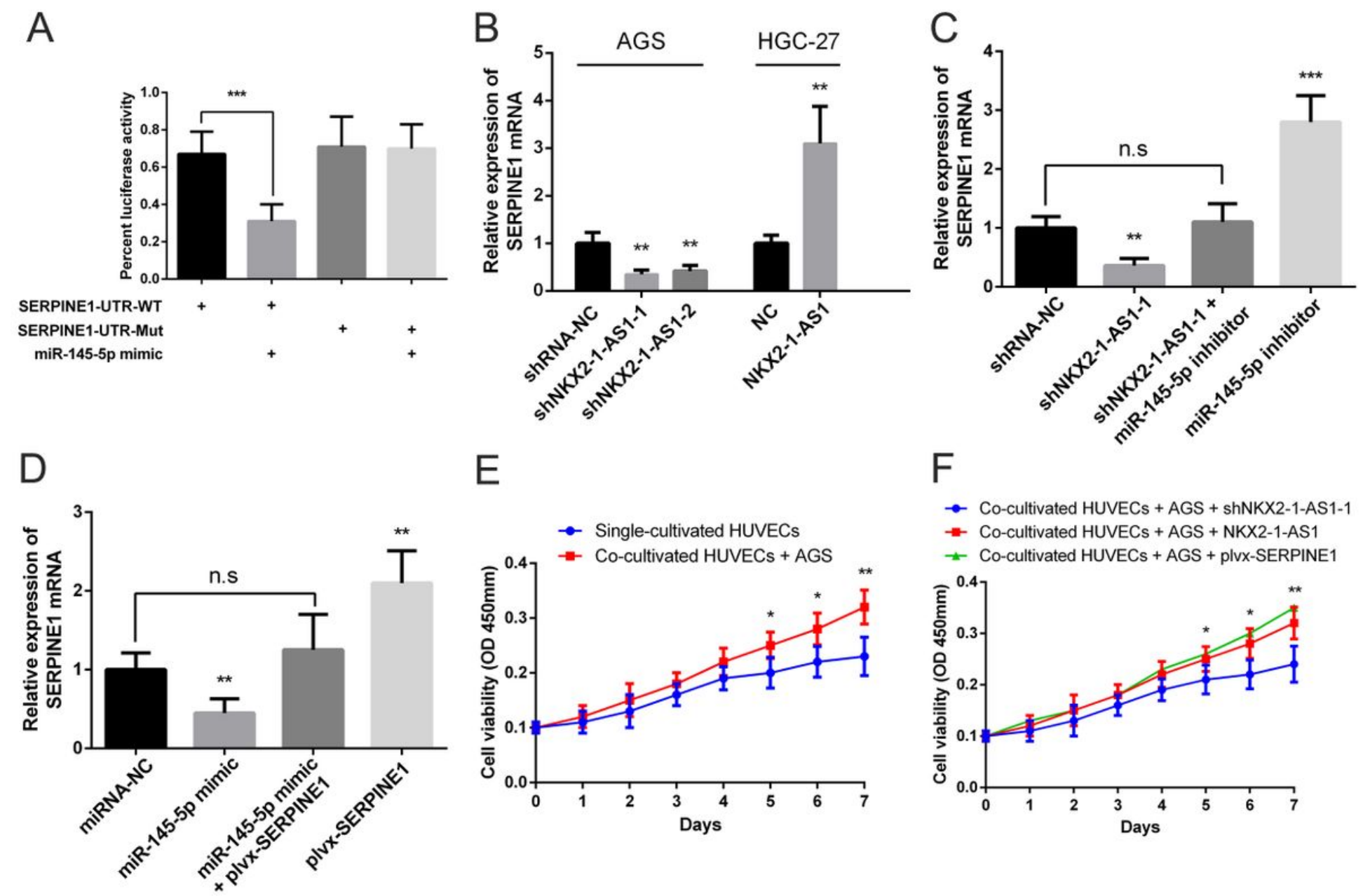

Figure 7

NKX2-1-AS1 promotes GC tissue angiogenesis by targeting miR-145-5p/SERPINE1. A Luciferase reporter assay in HEK-293FT cells co-transfected with wide type (WT) or mutated (Mut) SERPINE1 3'-UTR reporter vector and miR-145-5p 30 mimic. B Relative expression of SERPINE1 mRNA in AGS cells with NKX2-1AS1 knockdown or HGC-27 cells with NKX2-1-AS1 overexpression. C qRT-PCR was conducted to evaluate the mRNA expression of SERPINE1 gene in AGS cells following reduced expression of NKX2-1 -AS1 
and/or inhibition of miR-145-5p. D qRT-PCR was performed to access the mRNA expression of SERPINE1 gene in AGS cells following the ectopic expression of miR-145-5p and/or plvx-APC expression vector lacking the 3'-UTR. E The CCK-8 experiment was used to detect the cell proliferation activity of HUVECs cells when cultured alone and co-cultured with AGS cells. F NKX2-1-AS1 knocked down or overexpressed or SERPINE1 overexpressed AGS cells were co-cultured with HUVECs cells, CCK-8 experiment was used to detect HUVECs cell proliferation activity.
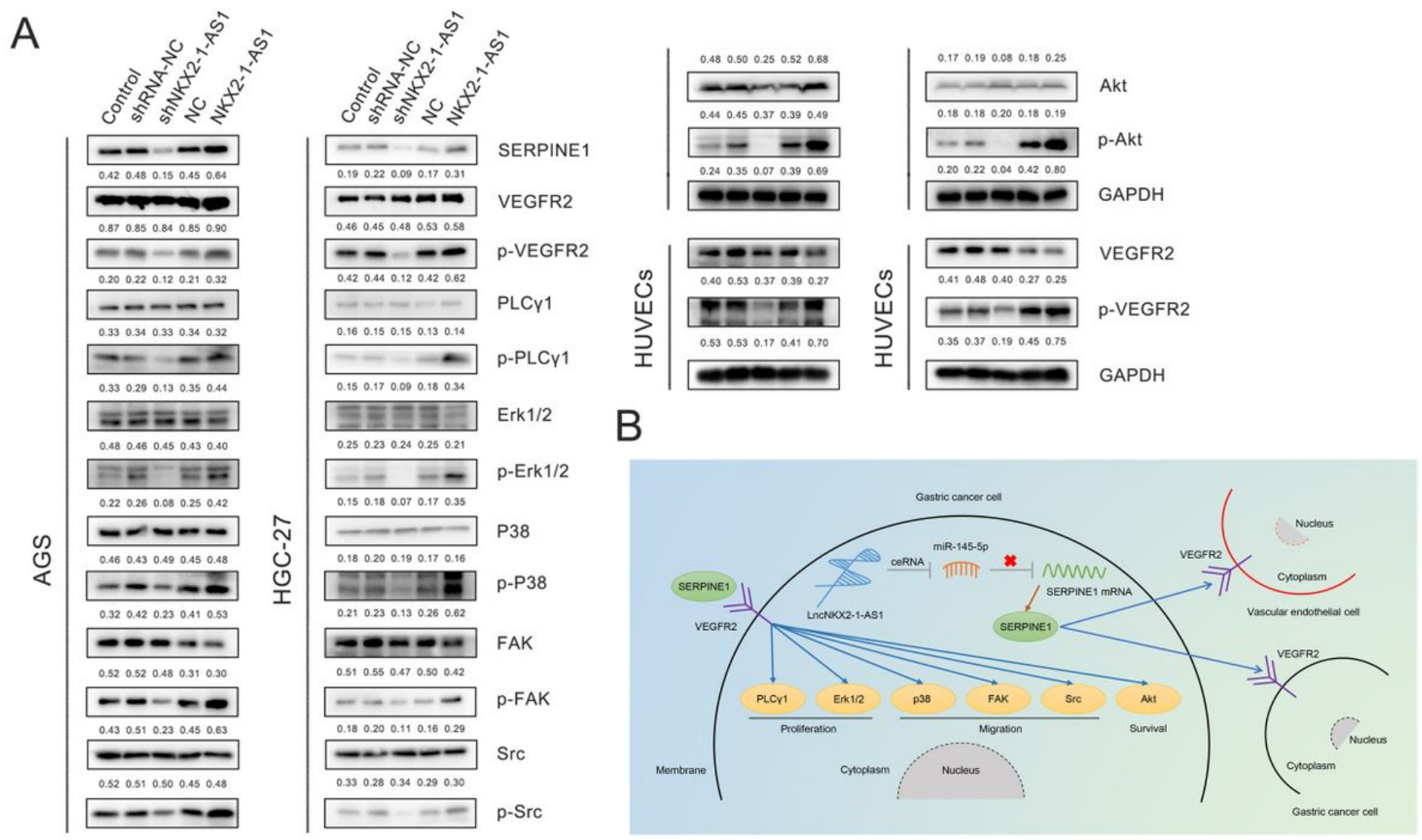

\section{Figure 8}

1-AS1/ miR-145-5p/ SERPINE1 axis positively regulates GC metastasis, invasion, and angiogenesis via the VEGFR-2 signaling pathway. A Immunoblot assay of SERPIN1, VEGFR-2, p-VEGFR-2, PLC- $\lambda$, p-PLC- $\lambda$, Erk1/2, p-Erk1/2, P38, p-P38, FAK, p-FAK, Src, p-Src, Akt, and p-Akt proteins in AGS cells transfected with shNKX2-1-AS1-1 and/or NKX2-1-AS1. Numbers showed quantification of relative protein amount. GAPDH was used as an internal control. After HUVECs cells were co-cultured with corresponding AGS cells for 7 days, the content of VEGFR-2 and p-VEGFR-2 protein was detected. GAPDH serves as an internal reference. B Schematic diagram of the regulatory mechanism of NKX2-1-AS1/miR-145$5 p / S E R P I N E 1$ axis in the promotion of GC proliferation, metastasis and angiogenesis.

\section{Supplementary Files}

This is a list of supplementary files associated with this preprint. Click to download. 
- TableS1.docx

- TableS1.docx

- FigS1.jpg

- FigS1.jpg

- table1.pdf

- table1.pdf 